\title{
Guidelines for clinical evaluation of chronic kidney disease
}

\author{
AMED research on regulatory science of pharmaceuticals and medical devices
}

\begin{abstract}
Eiichiro Kanda ${ }^{1,2} \cdot$ Naoki Kashihara $^{2} \cdot$ Kunihiro Matsushita $^{3} \cdot$ Tomoko Usui $^{4} \cdot$ Hirokazu Okada $^{5} \cdot$ Kunitoshi Iseki $^{6}$. Kenichi Mikami ${ }^{7} \cdot$ Tetsuhiro Tanaka $^{8} \cdot$ Takashi Wada $^{9} \cdot$ Hirotaka Watada $^{10} \cdot$ Kohjiro Ueki $^{11} \cdot$ Masaomi Nangaku $^{8}$. Research Working Group for Establishing Guidelines for Clinical Evaluation of Chronic Kidney Disease
\end{abstract}

Published online: 13 July 2018

(c) The Author(s) 2018

Keywords Surrogate endpoint $\cdot$ Glomerular filtration rate $\cdot$ End-stage renal disease $\cdot$ Dialysis $\cdot$ Chronic kidney disease $\cdot$ Proteinuria $\cdot$ Guideline

\section{Introduction}

In the clinical phase of drug development, it is important to establish appropriate endpoints. In general, to verify hard endpoints such as death, many subjects need to be studied over a long period of time, resulting in a large-scale clinical trial. To promote the early development of drugs, it is essential to establish methods for evaluating the clinical usefulness of treatment drugs by predicting long-term prognosis utilizing methods such as appropriate surrogate endpoints. In the field of nephrology, treatment of chronic diseases such as chronic kidney disease (CKD) and diabetic kidney disease

In 2018, Japanese Society of Nephrology established the Research Working Group for Establishing Guidelines for Clinical Evaluation of CKD, which published "Guidelines for Clinical Evaluation of Chronic Kidney Disease" in the Japanese Journal of Nephrology, 2018, vol. 60, issue 2, 67-100. This is the English version of that report. Chairman: Naoki Kashihara.

Masaomi Nangaku

mnangaku-tky@umin.ac.jp

1 Medical Science, Kawasaki Medical School, Okayama, Japan

2 Department of Nephrology and Hypertension, Kawasaki Medical School, Okayama, Japan

3 Department of Epidemiology, Johns Hopkins Bloomberg School of Public Health, Maryland, USA

4 Department of Pharmacoepidemiology, Graduate School of Medicine and Public Health, Kyoto University, Kyoto, Japan

5 Department of Nephrology, Saitama Medical University, Saitama, Japan
(DKD) is not satisfactory, and demands for the development of new drugs are strong. In Europe and the US, the use of surrogate endpoints for such diseases is being debated.

Based on this situation, the "Research Working Group for Establishing Guidelines for Clinical Evaluation of CKD" was set up as a research funded by the Research on Regulatory Science of Pharmaceuticals and Medical Devices of the Japan Agency for Medical Research and Development (AMED). In this program, the Japanese Society of Nephrology and Japanese Society of Diabetes worked together to establish guidelines for the clinical evaluation of treatment drugs for CKD, in collaboration with the Pharmaceuticals and Medical Devices Agency, analyzed data of Japanese cohorts. The aim of the project is to enable appropriate and prompt approval review of newly developed drugs without discrepancy from international criteria based on evidence for Japanese CKD patients.

6 Clinical Research Support Center, Tomishiro Central Hospital, Okinawa, Japan

7 Office of New Drug I, Pharmaceuticals and Medical Devices Agency (PMDA), Tokyo, Japan

8 Division of Nephrology and Endocrinology, Graduate School of Medicine, The University of Tokyo, 7-3-1 Hongo, Bunkyo-ku, Tokyo 113-8655, Japan

9 Department of Nephrology and Laboratory Medicine, Kanazawa University, Ishikawa, Japan

10 Department of Metabolism and Endocrinology, Juntendo University Graduate School of Medicine, Tokyo, Japan

11 Diabetes Research Center, National Center for Global Health and Medicine, Tokyo, Japan 
In this study, we established the guidelines of appropriate endpoints for the final evaluation of the effectiveness of newly developed drugs for CKD. In the study and development of drugs, specific study designs and the selection of biomarkers as endpoints in Phase 2 studies are important, but these were not analyzed or reviewed in this study.

\section{Preparation procedure}

The progress of the establishment of this guideline is summarized below. Throughout the whole program, discussions and exchange of opinions were frequently carried out by email. Related sessions were also set up during the various meetings of the Japanese Society of Nephrology to provide opportunities for the exchange of opinions.

Masaomi Nangaku participated in the KDIGO (Kidney Disease: Improving Global Outcomes) Controversies Conference "Challenges in the Conduct of Clinical Trials in Nephrology" held in Paris, France, between September 8 and 11,2016, to gather information on international trends and exchange opinions.

The first working group meeting was held on September 27,2016 . Prior to the meeting, preliminary discussions were carried out by email to verify planned analysis methods and role assignments of working group members. Thereafter, Eiichiro Kanda, Kunihiro Matsushita, and Tomoko Usui led a few analyses using data from Japanese studies such as a population-based cohort gathered by Kunitoshi Iseki and a Japanese CKD patient cohort study (CKD-JAC). In particular, the decline in estimate glomerular filtration rate (eGFR) and suitability of the surrogate endpoints of proteinuria/ albuminuria were reviewed. Furthermore, Eiichiro Kanda also conducted analysis of the Japanese DKD patient cohort study, as well as concurrently carried out systematic reviews of papers presented to date together with Tomoko Usui in a study led by Hirokazu Okada. Whether the results could be extrapolated to DKD was reviewed with the cooperation of Kohjiro Ueki, Hirotaka Watada, and Takashi Wada. The analytical work was overseen by Masaomi Nangaku, Naoki Kashihara, and Tetsuhiro Tanaka. Then, a rough draft of this guideline was prepared based on these analytical results with Kenichi Mikami, an open seminar was held on February 5, 2017 to gather extensive views, and based on the results, the second working group meeting was held on the same day. Vlado Perkovic (Australia) and Prabir Roy-Chaudhury (USA) were invited to the seminar and working group meeting as overseas experts to discuss differences from overseas trends. Based on the results of these meetings, the draft version of this guideline was prepared, announced during the 60th Annual Meeting of the Japanese Society of Nephrology held between May 26 and 28, 2017, and discussed with participants. Then between August 2 and 14, 2017, public comments were gathered widely through the Japanese Society of Nephrology and the Japan Diabetes Society, not limited to society members. The public comments received by email were then reviewed, and the third working group meeting was held on October 8, 2017, to finalize the guideline.

\section{Background}

CKD is a serious problem worldwide [1]. The number of patients in Japan is estimated to be 13.3 million, making it a national disease [2]. To prevent CKD patients from reaching end-stage renal disease (ESRD) which requires renal replacement therapy, there is a need to determine the progression factors of CKD, and establish treatment methods for protecting the kidneys. The development of treatments for CKD is an urgent task in terms of improving prognosis and Quality of Life of the patients, and from the perspective of economical healthcare. Also the development of treatments for DKD is particularly important, which is the number one cause for incipient dialysis. However, the development of treatments for CKD has been slow, and most treatments approved for CKD patients are targeting complications of CKD. In reality, the number of randomized control trials required for the approval of kidney disease treatments is very small compared to other areas [3]. The underlying reasons include absence of appropriate model animals due to the complicated pathophysiology of kidney disease and the difficulty of clinical trials [4].

To carry out appropriate clinical trials for CKD, it is essential to secure an adequate number of patients for outcome evaluation, prevent dropouts during the trial period, and carry out correct statistical analysis using endpoints that are suitable for evaluation. In the clinical trials of drug effectiveness evaluation of treatment drugs for CKD, hard endpoints such as renal death (dialysis or renal transplantation) or doubling of serum creatinine level have been used. To study this end point, there is a need to recruit many patients and follow up on them over a long time. This has made clinical trials difficult.

In the clinical trials of CKD, the outcomes unique to kidney are needless to say appropriate endpoints. Regarding setting mortality as an outcome, given that sensitivity and generalization feasibility are both low, it is rarely possible to appropriately use mortality as an endpoint of CKD clinical trials [5]. Since deaths are caused by various reasons, it is hard to associate direct reasons with CKD studied in clinical trials. For CKD, ESRD has been used as the true endpoint in various clinical studies. The definition of ESRD differs according to the research. It often includes implementation of dialysis, renal transplantation, and doubling of serum creatinine level. However, given the low incidence rate of events, long-term observation and large-scale sample 
size are required. For clinical studies to be carried out efficiently, the development of useful surrogate endpoints is indispensable.

According to the US Biomarkers Definitions Working Group consisting mainly of members of the National Institutes of Health (NIH), a biomarker is defined as "a characteristic that is objectively measured and evaluated as an indicator of normal biological processes, pathogenic processes, or pharmacologic responses to a therapeutic intervention." [6], while a surrogate endpoint is defined as "a biomarker that is intended to substitute for a clinical endpoint. A surrogate endpoint is expected to predict clinical benefit (or harm or lack of benefit or harm) based on epidemiologic, therapeutic, pathophysiologic, or other scientific evidence." [6]. Consequently, surrogate endpoints are biomarkers and must be strongly associated with true endpoints.

The criteria for the strength of a surrogate endpoint are defined by the International Conference on Harmonization of Technical Requirements for Registration of Pharmaceuticals for Human Use (ICH E9) as follows: (1) the biological plausibility of the relationship, (2) the demonstration in epidemiological studies of the prognostic value of the surrogate for the clinical outcome, and (3) evidence from clinical trials showing that treatment effects on the surrogate correspond to the effects on the clinical outcome [7].

If reliable surrogate endpoints are available, it may be possible to decrease the clinical study sample size and shorten the duration as well. Even for the evaluation of outcomes specific to renal disease using surrogate endpoints, the appropriate endpoints are expected to vary according to the speed of progress and clinical stage of the disease. KDIGO proposed the endpoints shown in Table 1 based on the results of the controversy conference held in 2016 [5].

To optimize the design and operation of clinical trials, the international working group meeting hosted by the National Kidney Foundation (NKF) and Food and Drug Administration (FDA) presented a series of papers reviewing the feasibility of using decrease in eGFR as surrogate endpoints of ESRD for clinical trials in 2014 [8-11]. The general conclusion of these papers supported 30-40\% decrease in eGFR as a surrogate endpoint of ESRD [12]. This conclusion was generally well received by researchers and drug regulatory authorities [13-15].

However, the papers of the NKF-FDA Working Group had a limitation of predominantly using research data from Europe and North America [8-11]. For instance, in a major paper from the Working Group using the data from the CKD Prognosis Consortium, only $0.5 \%$ of study population for ESRD were Asians [8]. Given the vast regional differences in the prevalence and incidence of ESRD (East Asian countries and regions such as Japan and Taiwan are ranked amongst the top in the world) $[1,16]$, as well as the fact that kidney disease pattern is unique in the Asian region (percentage of IgA nephropathy is relatively high, etc.) [17], the results from the NKF-FDA Working Group may not be simply extrapolated to Japanese. For Japanese CKD patients, a small study with 701 subjects demonstrated that a 30\% decrease in eGFR over 2 years was strongly related to ESRD risk [18], but large-scale studies are needed for definite conclusions. As clinical trial endpoints, the decrease in albuminuria/proteinuria is frequently used, but the validity of surrogate endpoints replacing suppression of progress to ESRD has yet to be reviewed adequately.

Thus, in this study, the Chronic Kidney Disease Japan Cohort (CKD-JAC), a representative CKD cohort in Japan, and a health checkup database of Okinawa Prefecture was analyzed in parallel. In addition, data from a randomized controlled trial (RCT) of Japanese patients with diabetic nephropathy were also analyzed. In addition, separate from the data analysis of these Japanese studies, systematic review on existing evidence was carried out, and the results were also reviewed to establish this guideline. The results from the analysis of those studies indicated that it may not be optimal to apply a single surrogate outcome to any situations. Thus, this guideline tried to leave some flexibility. The appropriate number of measurements of serum creatinine level for calculating eGFR is not indicated in this guideline, since this

Table 1 Renal disease endpoints proposed by KDIGO [5]

\begin{tabular}{|c|c|c|}
\hline & CKD progressing slowly & $\mathrm{CKD}^{\mathrm{a}}$ progressing fast \\
\hline $\begin{array}{l}\text { Early stage: CKD G1-G3a } \\
\left(\text { eGFR } \geq 45 \mathrm{ml} / \mathrm{min} \text { per } 1.73 \mathrm{~m}^{2}\right)\end{array}$ & $\begin{array}{l}\text { Slope of mGFR or eGFR or surrogate outcome }{ }^{\mathrm{b}} \text { or combinations of out- } \\
\text { comes }\end{array}$ & $\begin{array}{l}30-40 \% \text { decline in eGFR using } \\
\text { repeat measurements to rule } \\
\text { out transient acute effects }{ }^{c}\end{array}$ \\
\hline $\begin{array}{l}\text { Late stage: CKD G3b-G5 } \\
\left(\text { eGFR }<45 \mathrm{ml} / \mathrm{min} \text { per } 1.73 \mathrm{~m}^{2}\right)\end{array}$ & End-stage kidney disease or $30 \% \sim 40 \%$ decline in $\mathrm{eGFR}^{\mathrm{c}}$ & $\begin{array}{l}\text { End-stage kidney disease or } \\
\text { doubling of serum creatinine } \\
\text { level (or } 40 \% \sim 57 \% \text { decline in } \\
\text { eGFR) }\end{array}$ \\
\hline
\end{tabular}

${ }^{\mathrm{a}}$ For example, in patients with macroalbuminuria

${ }^{\mathrm{b}}$ Surrogates may include measures of activity of disease (e.g., in lupus nephritis) or kidney structure (e.g., in adult polycystic kidney disease)

${ }^{\mathrm{c}}$ The added value of eGFRs outside the routine study visit schedule has not yet been demonstrated and they may be unnecessary 
cannot be calculated based on evidence. If we focus on a very specific population, it may be possible to make the descriptions more specific in this guideline. However, since it will not be possible to establish evidence due to the decrease in sample size to be reviewed in this case, the following descriptions were added. Given the possibility that appropriate endpoints may differ according to the research, there is a need to set appropriate surrogate endpoints when designing studies, taking into account the characteristics of subjects, underlying disease, and test drug. DKD is an important cause of CKD. In Japan, DKD is the most common cause of incident dialysis since 1998. In this study, it was specifically verified if surrogate endpoints can also be applied for DKD. For DKD, the management of blood glucose and blood pressure is obviously important [19]. There is a need to raise the awareness that even during the clinical trial of new drugs, their strict management is a major prerequisite.

\section{Relation between decrease in estimated glomerular filtration rate in Japanese CKD patients and risks of end-stage renal disease: analysis of CKD-JAC}

\section{Methods}

\section{Study population}

In this study, we investigated changes in eGFR and subsequent risk of ESRD using the data of the Chronic Kidney Disease Japan Cohort (CKD-JAC), a representative CKD cohort study in Japan. Details of the results of that cohort study were reported in a peer-reviewed journal, Kidney International (November 2016 issue). This section provides a key summary of that article [20].

The CKD-JAC was conducted to investigate risk factors for CKD progression in Japan, and its design and data gathering method have been presented in other papers [21, 22]. It included 2966 Japanese adults (age 20-75 years with an eGFR of $10-59 \mathrm{ml} / \mathrm{min} / 1.73 \mathrm{~m}^{2}$ ) from 17 clinical facilities throughout Japan from April 2007 to December 2008 [22]. The following subjects were excluded: polycystic kidney disease, HIV infection, liver cirrhosis, organ transplantation, chronic dialysis, and pregnancy. At baseline, serum creatinine level, urinary albumin level, and medical history were investigated in addition to basic characteristics such as age. The patients with serum creatinine level within 1 year (2410 subjects) and 2 years (2079 subjects) measured at a central laboratory were enrolled in this analysis.

\section{Change in eGFR}

Serum creatinine level was measured at LSI Medience Corporation (Tokyo, Japan) using an enzymatic method. The same machine and reagent were used for the measurements during the entire study period of the CKD-JAC. eGFR was calculated using an eGFR equation, which was developed by Matsuo and others, for the Japanese population [23]. Like the previous studies by the NKF-FDA Working Group, the changes in eGFR were calculated as follows:

(last eGFR at baseline period - first available eGFR)/(first available eGFR) $\times 100[8,10]$.

Although the NKF-FDA Working Group evaluated the changes in eGFR over 1-3 years, because the median follow-up period of CKD-JAC was 3.9 years, the current study investigated the changes in eGFR over 1 and 2 years and the following risks of ESRD. The subsequent median follow-up period was 2.9 and 2.0 years, respectively.

\section{ESRD (end-stage renal disease)}

The subjects were followed up every 6 months through clinical visits to the participating clinical facilities. Phone interviews were also carried out when needed. The primary endpoint was ESRD defined as the start of hemodialysis or peritoneal dialysis or kidney transplantation until March 31, 2013. Subjects who did not develop ESRD were followed up until the last clinical visit, death, or March 31, 2013, whichever came first.

\section{Covariates}

Age, gender, and smoking status were based on information reported by the subjects. Diabetes mellitus (DM) was defined as hemoglobin A1c $\geqq 6.5 \%$, history of diagnosis of $\mathrm{DM}$, or use of glucose-lowering medications. A history of cardiovascular disease was defined as a history of coronary disease, stroke, or heart failure. A triple measurement average of blood pressure was analyzed. Serum total cholesterol level, hemoglobin level, urine albumin, and creatinine levels were measured at LSI Medience Corporation. Serum total cholesterol levels were measured using an enzymatic method and hemoglobin level by the SLS-Hb method. Urinary albumin and creatinine levels were measured with the trypsin inhibitor activity and enzymatic methods, respectively.

\section{Statistical analysis}

Baseline and clinical characteristics of the subjects were compared between four groups of changes in eGFR [ $\leq-53 \%$ (doubling of serum creatinine level), $>-53$ to $-30 \%,>-30-0 \%$, and $>0 \%$ (which is increase in eGFR)]. The Cox proportional hazards model was then used to estimate the adjusted hazard 
ratios (aHRs) of ESRD to change in eGFR using spline terms with knots at $-53,-25,-10,10$, and $25 \%$. In our primary analysis, such as the analysis done by the NKF-FDA Working Group, the Cox proportional hazards models were adjusted for age, gender, systolic blood pressure, serum total cholesterol level, DM, history of cardiovascular disease, and first baseline eGFR. For sensitivity analysis, urinary albumin and hemoglobin levels were additionally included in the model. We also carried out analysis on subgroups by age, gender, and causes of CKD at baseline. Whether there were statistical interactions was evaluated by comparing models with and without interactions between the subgroups and changes in eGFR using likelihood ratio tests. We also predicted the risks of ESRD by change in eGFR and baseline eGFR at 1-3 years after the baseline period. At this time, other covariates were set as follows: age, 60 years; male, gender; systolic blood pressure, $130 \mathrm{~mm}$ $\mathrm{Hg}$; serum total cholesterol level, $5 \mathrm{mmol} / \mathrm{l}$; and no history of $\mathrm{DM}$ or cardiovascular disease. A competing risk model was used treating death as a competing endpoint.

\section{Results}

The characteristics of the subjects are summarized in Tables 2 and 3. Out of the 2410 subjects whose eGFR was measured 1 year later again after the baseline eGFR, 1.4\% (34 subjects) showed more than 53\% decrease. On the other hand, $4.3 \%$ (104 subjects) and 9.7\% (233 subjects) showed changes in eGFR of more than $40 \%$ and more than $30 \%$, respectively (Fig. 1a). And, 4.2\% (87 subjects), 10.9\% (227 subjects), and $19.3 \%$ (401 subjects) of 2079 subjects showed more than 53, 40, and 30\% decrease in eGFR over 2 years, respectively (Fig. 1b).
The average change in eGFR (SD) was $-13 \%$ (22\%) over 2 years and $-7 \%$ (19\%) over 1 year. Compared to the group with moderate changes in eGFR $(>-30-0 \%$ change), those with more than $30 \%$ decrease in eGFR were mainly female, current smokers, and had a history of DM or cardiovascular disease. Moreover, many with 53-30\% decrease in eGFR had high serum total cholesterol level, high blood pressure, high urinary albumin-to-creatinine ratio, low eGFR, and low hemoglobin level (Tables 2, 3).

Out of the 2410 participants reviewed for change in eGFR over 1 year and risk of ESRD, ESRD was observed in 498 subjects $(20.7 \%)$ during a median follow-up of 2.9 years. In the analysis of change in eGFR over 2 years, ESRD was observed 365 subjects (17.6\%) out of 2079 subjects during a median follow-up of 2.0 years. The decrease in eGFR over 1 and 2 years was strongly related to the risk of developing ESRD (Fig. 2). The relative risk of developing ESRD with the 53\% decrease in eGFR over 1 and 2 years was 21and 17-times (aHR 20.72 [95\% confidence interval (CI), 14.27-30.09] and 17.27 [11.80-25.30]), respectively. Both the 40 and $30 \%$ decreases in eGFR were also strongly related to ESRD risk. For the 1-year change, aHR was 9.61 (95\% CI, 7.42-12.46) with the $40 \%$ decrease in eGFR and 5.32 (4.14-6.85) with the $30 \%$ decrease in eGFR. For the 2-year change, it was $6.53(4.70-9.05)$ with the $40 \%$ decrease in eGFR and 3.09 (2.15-4.42) with the 30\% decrease in eGFR. Although increased risk of ESRD was associated with the increase in eGFR in the 1-year (Fig. 2a), the same tendency was not found in the 2-year analysis (Fig. 2b).

Following the analyses of the primary analysis, several sensitivity analyses were also examined. First, albuminuria and hemoglobin levels were additionally adjusted,

Table 2 Baseline characteristics of subjects with percentage change in eGFR over 1 year

\begin{tabular}{|c|c|c|c|c|}
\hline & \multicolumn{4}{|c|}{ Percentage change in eGFR over 1 year $(\%)$} \\
\hline & $\leq-53$ & $>-53$ to $\leq-30$ & $>-30$ to $\leq 0$ & $>0$ \\
\hline No. of subjects & 34 & 199 & 1375 & 802 \\
\hline ESRD event, $n(\%)$ & $30(88.2)$ & $133(66.8)$ & $256(18.6)$ & $79(9.9)$ \\
\hline Age, mean (SD), age & $60.1(11.8)$ & $61.3(11.4)$ & $60.9(11.5)$ & $61.5(11.1)$ \\
\hline Female, $n(\%)$ & $21(61.8)$ & $135(67.8)$ & $840(61.1)$ & $501(62.5)$ \\
\hline Smoker, $n(\%)$ & $3(8.8)$ & $39(19.6)$ & $202(14.7)$ & $102(12.7)$ \\
\hline $\mathrm{DM}, n(\%)$ & $18(52.9)$ & $107(53.8)$ & $498(36.2)$ & $268(33.4)$ \\
\hline History of cardiovascular disease, $n(\%)$ & $6(17.6)$ & $53(26.6)$ & $285(20.7)$ & $190(23.7)$ \\
\hline Total cholesterol, mean (SD), mmol/L & $5.5(1.8)$ & $5.1(1.3)$ & $5(1.1)$ & $4.9(1.1)$ \\
\hline $\mathrm{SBP}$, mean (SD), mmHg & $140.1(21.5)$ & $138.1(18.6)$ & $132.1(17.9)$ & $127.6(17.7)$ \\
\hline DBP, mean (SD), mmHg & $76.6(10.2)$ & $76.9(12.3)$ & $76.9(12)$ & $74.7(11.5)$ \\
\hline Use of antihypertensive drug, $n(\%)$ & $34(100)$ & 197 (99) & $1316(95.7)$ & $741(92.4)$ \\
\hline eGFR, mean (SD), ml/min/1.73 m² & $22.3(10.7)$ & $20(12.2)$ & $28.2(14.9)$ & $28.5(14.8)$ \\
\hline $\begin{array}{l}\text { Albuminuria, median (interquartile } \\
\text { range), } \mathrm{Alb} \mathrm{mg} / \mathrm{gCr}\end{array}$ & $2663.3(1205.5,4070.9)$ & $1688.7(849.4,3241.2)$ & $627.4(180.2,1298.2)$ & $186.7(41.4,559.5)$ \\
\hline Hemoglobin, mean (SD), g/dL & $10.5(1.4)$ & $11.1(1.6)$ & $12.2(1.8)$ & $12.2(1.8)$ \\
\hline
\end{tabular}


Table 3 Baseline characteristics of subjects with percentage change in eGFR over 2 years

\begin{tabular}{|c|c|c|c|c|}
\hline & \multicolumn{4}{|c|}{ Percentage change in eGFR over 2 years (\%) } \\
\hline & $\leq-53$ & $>-53$ to $\leq-30$ & $>-30$ to $\leq 0$ & $>0$ \\
\hline No. of subjects & 87 & 314 & 1153 & 525 \\
\hline ESRD event, $n(\%)$ & $71(81.6)$ & $154(49)$ & $104(9)$ & $36(6.9)$ \\
\hline Age, mean (SD), age & $59.9(10.6)$ & $61.3(11.6)$ & $60.7(11.2)$ & $60.9(11.4)$ \\
\hline Female, $n(\%)$ & $54(62.1)$ & $210(66.9)$ & $670(58.1)$ & $332(63.2)$ \\
\hline Smoker, $n(\%)$ & $16(18.4)$ & $56(17.8)$ & $153(13.3)$ & $65(12.4)$ \\
\hline $\mathrm{DM}, n(\%)$ & $44(50.6)$ & $145(46.2)$ & $378(32.8)$ & $173(33)$ \\
\hline History of cardiovascular disease, $n(\%)$ & $25(28.7)$ & $82(26.1)$ & $221(19.2)$ & $117(22.3)$ \\
\hline Total cholesterol, mean (SD), mmol/L & $5.2(1.4)$ & $5.1(1.1)$ & $5(1.1)$ & $5(1.1)$ \\
\hline $\mathrm{SBP}$, mean (SD), mmHg & $137.8(19.7)$ & $136.5(16.5)$ & $130.5(17.3)$ & $125.5(17.8)$ \\
\hline DBP, mean (SD), mmHg & $76.7(12.2)$ & $77.3(12)$ & $76.5(11.4)$ & $74.4(11.9)$ \\
\hline Use of antihypertensive drug, $n(\%)$ & $86(98.9)$ & $312(99.4)$ & $1092(94.7)$ & $481(91.6)$ \\
\hline eGFR, mean (SD), $\mathrm{ml} / \mathrm{min} / 1.73 \mathrm{~m}^{2}$ & $24.4(11.1)$ & $22.2(11.2)$ & $30(14.9)$ & $30(15)$ \\
\hline $\begin{array}{l}\text { Albumin-to-creatinine ratio, median (inter- } \\
\text { quartile range), } \mathrm{mg} / \mathrm{g}\end{array}$ & $1602.8(956.7,3262.3)$ & $1292.4(604,2291.6)$ & $482.1(130.1,1049.8)$ & $128.3(29.9,408.9)$ \\
\hline Hemoglobin, mean (SD), g/dL & $11.2(1.7)$ & $11.6(1.6)$ & $12.3(1.7)$ & $12.4(1.9)$ \\
\hline
\end{tabular}

a

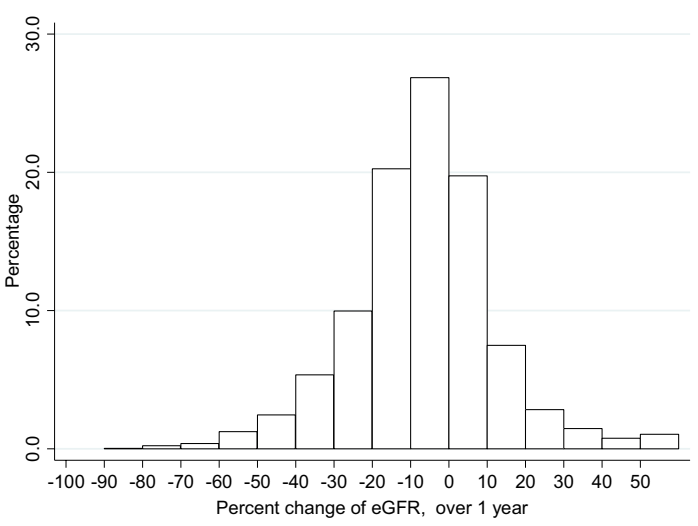

b

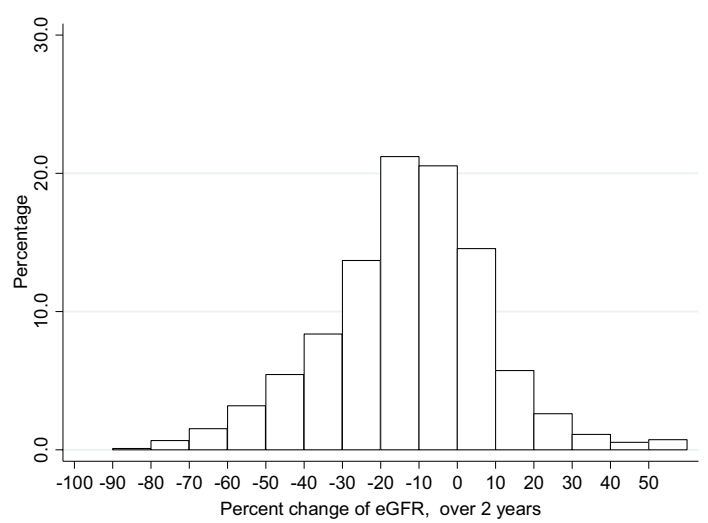

Fig. 1 Distribution of percentage changes in eGFR. a Percentage change in eGFR over 1 year. b Percentage change in eGFR over 2 years. Percentage change in eGFR was calculated as follows: (last eGFR at baseline period - first available eGFR)/(first available eGFR) $\times 100$

but similar results were obtained with regard to the relation between change in eGFR and ESRD risks (Fig. 3). Next, subgroup analyses were conducted by age, gender, and causes of CKD (diabetic nephropathy, hypertensive renal disease, glomerular disease, etc.) (Figs. 4, 5, 6). The association between change in eGFR and ESRD risk was found to be significantly stronger in men than in women (Interaction, $P<0.001$, Fig. 5). Basically, the tendency for this strong association between change in eGFR and ESRD risk was the same in all the subgroups tested.

Finally, Figs. 7 and 8 summarize the results of predicting eGFR at baseline and change in eGFR, and the risks of developing ESRD 1-3 years after the baseline period. When the baseline eGFR was $50 \mathrm{ml} / \mathrm{min} / 1.73 \mathrm{~m}^{2}$ (CKD Stage G3a), the risk of ESRD in 3 years was predicted to be $4-5 \%$ even for the decrease in eGFR of 53\% over 1 and 2 years. However, when baseline eGFR was $35 \mathrm{ml} / \mathrm{min} / 1.73 \mathrm{~m}^{2}$ (CKD Stage G3b), the risk was 35 and $30 \%$ for decrease in eGFR of $53 \%$ over 1 and 2 years, respectively. The risk was 19 and $11 \%$ for decrease in eGFR over 1 year and $14 \%$ and $7 \%$ for decrease in eGFR over 2 years of 40 and 30\%, respectively. When baseline eGFR was $25 \mathrm{ml} / \mathrm{min} / 1.73 \mathrm{~m}^{2}$ (CKD Stage G4), the ESRD risk in 3 years was predicted to be 86,61 , and $42 \%$ for change in eGFR over 1 year of 53,40 and $30 \%$, respectively $(77,45$, and $26 \%$ for change in eGFR over 2 years of 53,40 and $30 \%$, respectively). 


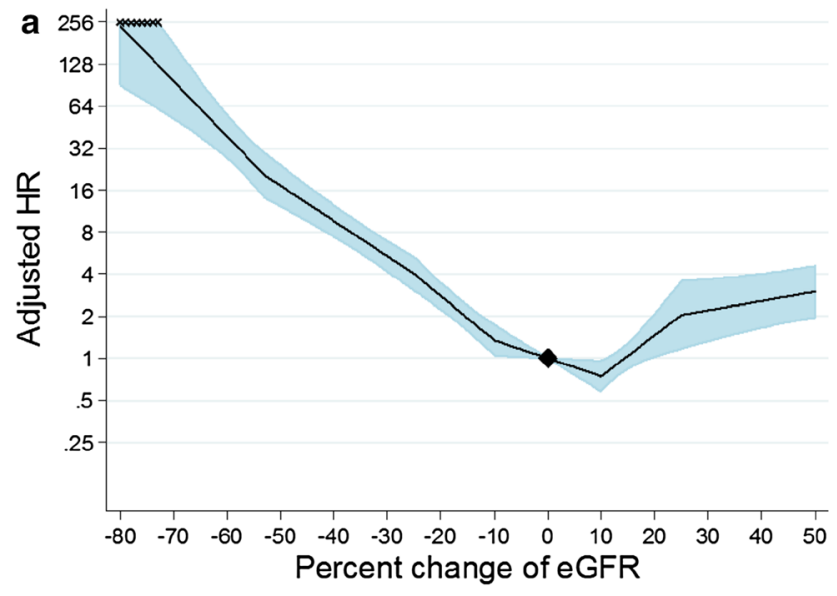

Fig. 2 Relationships between percentage changes in eGFR and risk of ESRD. a Percentage change in eGFR over 1 year. b Percentage change in eGFR over 2 years. The hazard ratio of ESRD according to

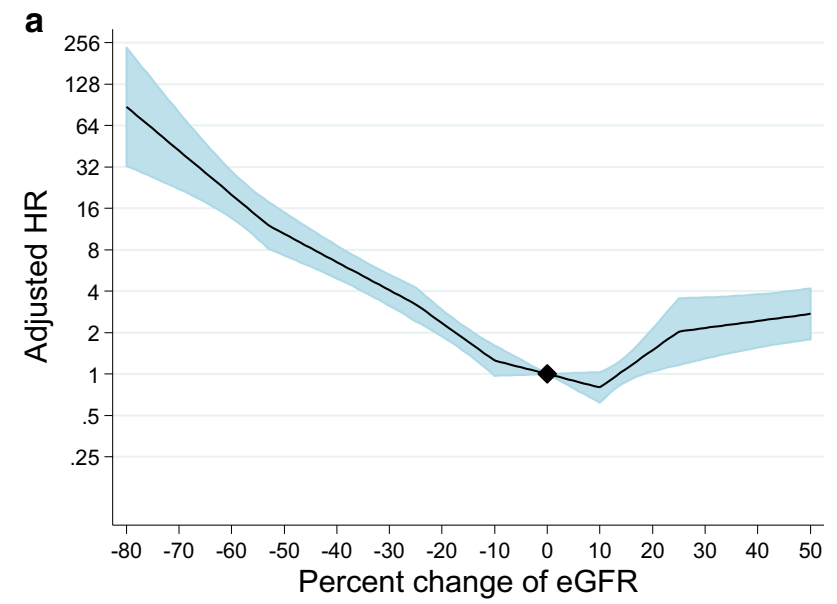

Fig. 3 Relationships between percentage changes in eGFR and risk of ESRD. a Percentage change in eGFR over 1 year. b Percentage change in eGFR over 2 years. The hazard ratio of ESRD according to percentage change in eGFR was adjusted for age, gender, DM, history

\section{Discussion}

In this study, changes in eGFR over 1 and 2 years were confirmed to be strongly associated with subsequent ESRD risk in Japanese CKD patients regardless of adjustments for confounders and baseline eGFR. Given the fact that doubling of serum creatinine level has already been accepted as a surrogate endpoint of ESRD, 53\% decrease in eGFR over 1 and 2 years showed 17-21 times higher risk of ESRD than $0 \%$ change in eGFR. Although not as high as the 53\% decrease, 40 and 30\% decrease in eGFR over 1 and 2 years led to significantly high risk of ESRD (7-10 times and 3-5 times, respectively). Since 30\% decrease in eGFR or greater was found in five to seven times more subjects compared to

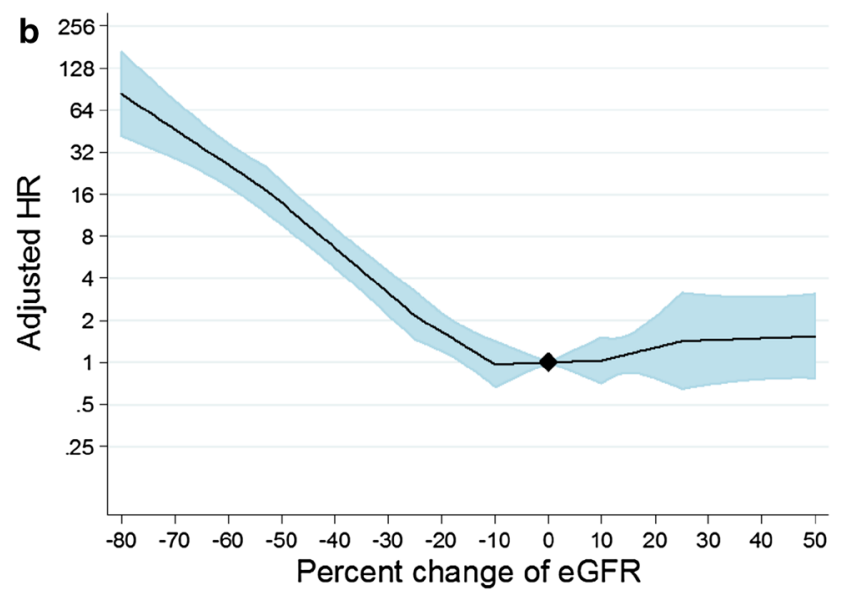

percentage change in eGFR was adjusted for age, gender, DM, history of cardiovascular disease, systolic blood pressure, serum total cholesterol level, and first available eGFR

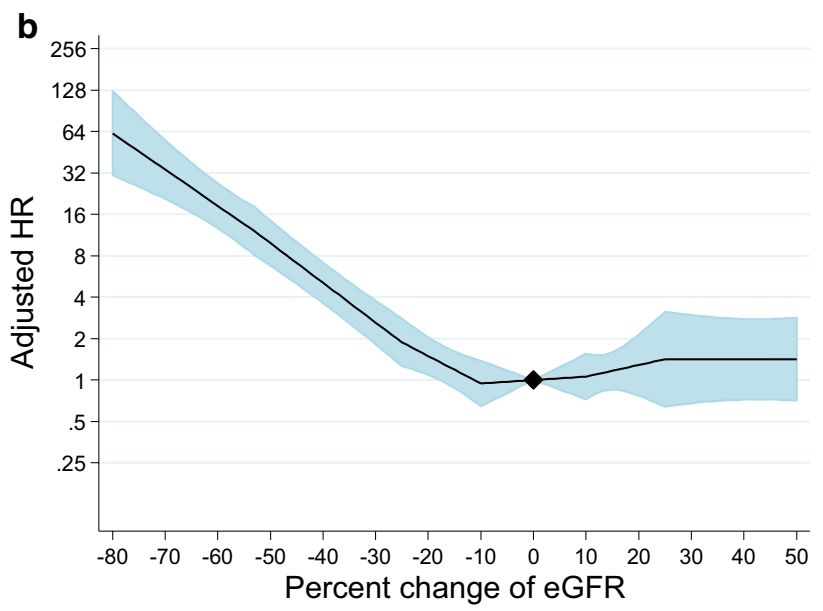

of cardiovascular disease, systolic blood pressure, serum total cholesterol level, and first available eGFR, as well as urinary albumin-tocreatinine ratio and hemoglobin levels

53\% decline, this is an important finding when the number of events and statistical power are estimated in the clinical trials. Basically, the decrease in eGFR was related to the increase in ESRD regardless of age, gender, and causes of CKD.

In this analysis, although the association between decrease in eGFR and ESRD risk was found to be continuous in our study (Fig. 2), it is not easy to narrow down thresholds for decrease in eGFR to a surrogate endpoint. Bringing the threshold for decrease in eGFR closer to the doubling of serum creatinine level leads to the identification of cases with very high ESRD risk, but the number of events will be limited. On the other hand, selecting a more moderate decrease in eGFR as the threshold enables more cases to 

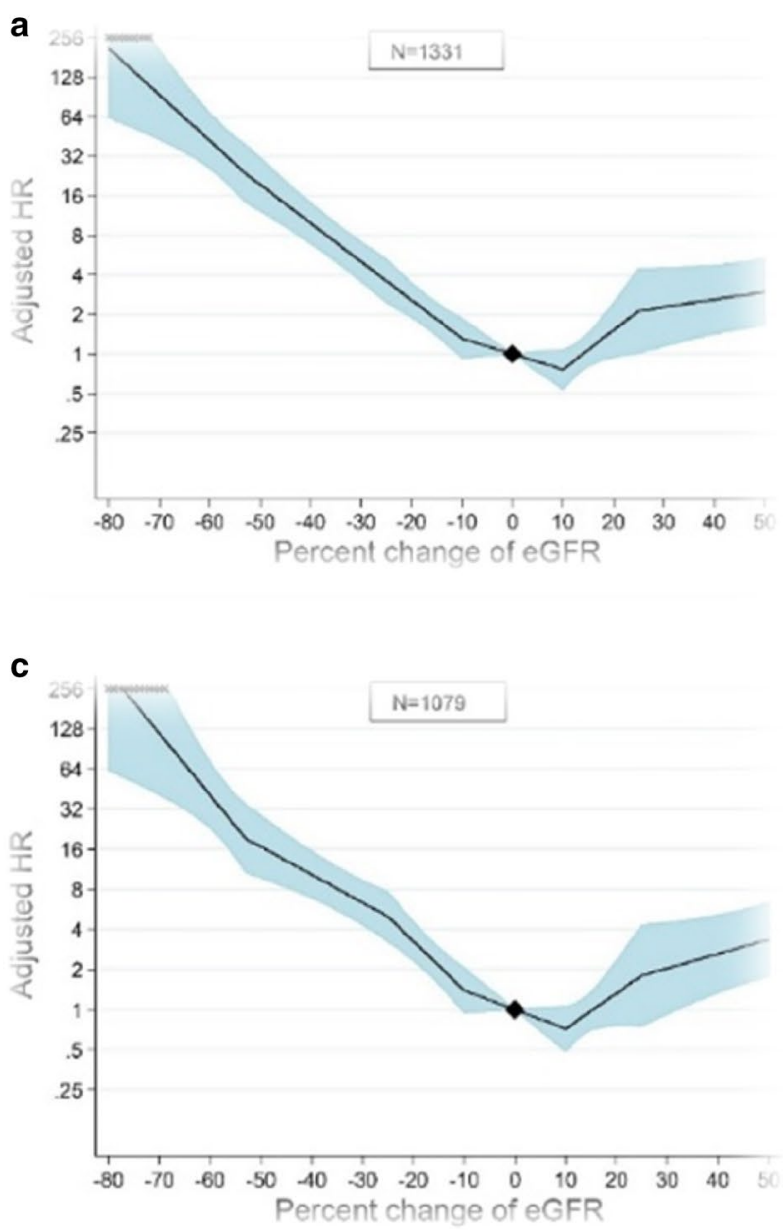

Fig. 4 Relationships between percentage changes in eGFR and risk of ESRD by age. a Percentage change in eGFR over 1 year (less than 65 years old). b Percentage change in eGFR over 2 years (less than 65 years old). c Percentage change in eGFR over 1 year (65 years old or older). d Percentage change in eGFR over 2 years (65 years old

be taken as events, but this means that cases with relatively low ESRD risk will also be included in the events. However, 30-40\% decrease in eGFR was proposed as a surrogate endpoint of CKD progression by the NKF-FDA Working Group, based on assessment of the relation between change in eGFR and ESRD risk, differences in the evaluation of treatment results using ESRD and surrogate endpoints, and numerous simulation analyses, and this is an important fact. Where there are no acute effects of new drugs on eGFR or small protective effects in the subjects with GFR less than $30 \mathrm{ml} / \mathrm{min} / 1.73 \mathrm{~m}^{2}, 30 \%$ decrease in eGFR was proposed as an appropriate surrogate endpoint by the NKF-FDA Working Group [12]. For drugs whose positive acute effects continue over a long term, the use of change in eGFR of more than $30 \%$ as a surrogate endpoint is also evaluated as suitable. The group also stated that the surrogate endpoint of $40 \%$ is adequate for a wide range of acute effects and
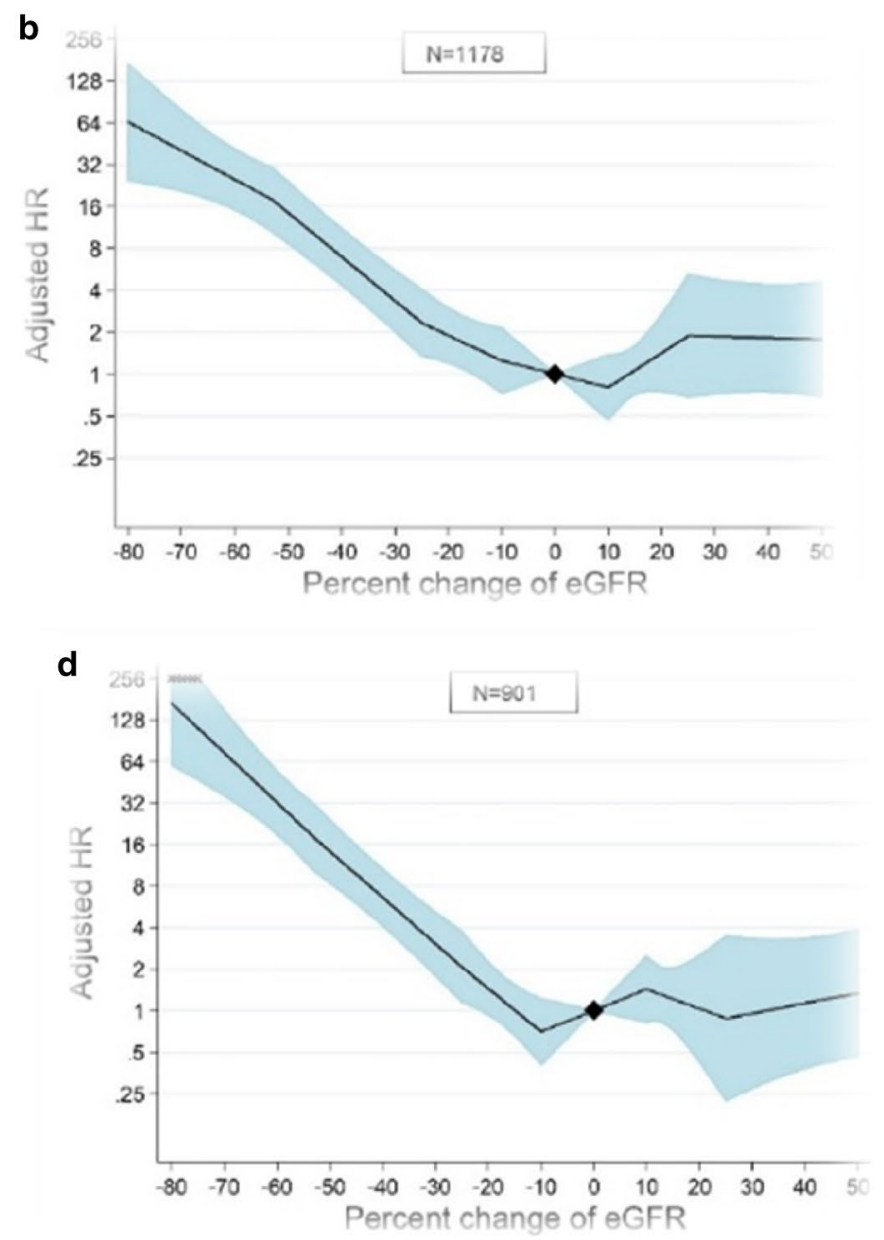

or older). The hazard ratio of ESRD according to percentage change in eGFR was adjusted for age, gender, DM, history of cardiovascular disease, systolic blood pressure, serum total cholesterol level, and first available eGFR

baseline eGFRs [8]. The threshold for surrogate endpoints cannot be determined from our study alone, but our results and the statement of the group suggest that more moderate decrease in eGFR than $53 \%$ may be an appropriate surrogate endpoint of CKD progression in Japanese CKD patients.

In this study, decrease in eGFR was found to be strongly related to ESRD risk statistically in men than in women. This result may be consistent with previous studies showing that GFR decline in men is faster than in women [24, 25]. However, controversy surrounds this issue of the relation of gender difference to the decrease in GFR [26]. Still, it is an important fact that the decrease in eGFR and ESRD risk was found to be consistently associated in both men and women. Consistent association was more or less found across subgroups by age and causes of CKD including diabetic nephropathy. Given that DM is the leading cause of ESRD in developed countries and is an important disease 

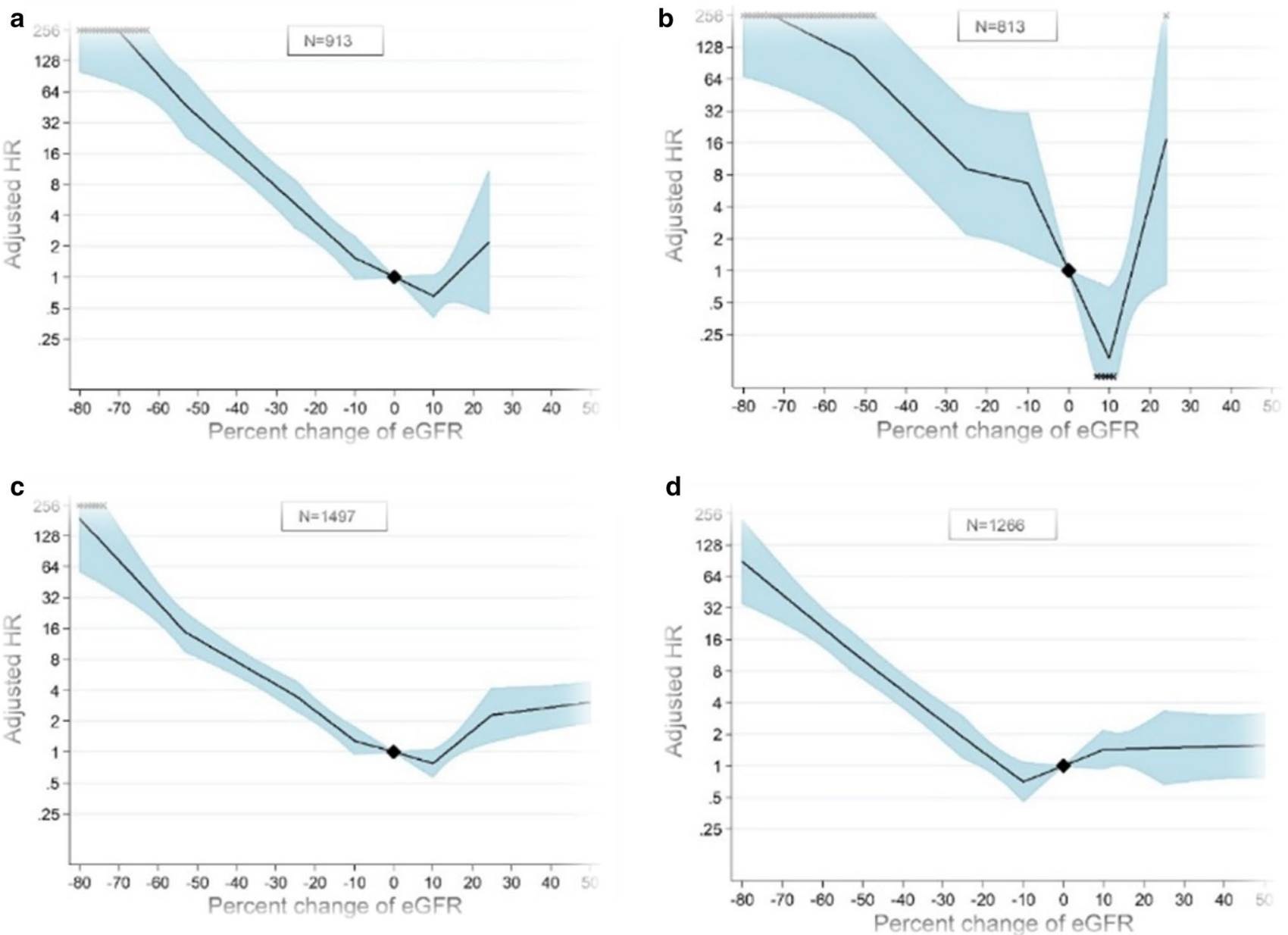

Fig. 5 Relationships between percentage changes in eGFR and risk of ESRD by gender. a Percentage change in eGFR over 1 year (male). $\mathbf{b}$ Percentage change in eGFR over 2 years (male). c Percentage change in eGFR over 1 year (female). d Percentage change in eGFR over

2 years (female). The hazard ratio of ESRD according to percentage change in eGFR was adjusted for age, gender, DM, history of cardiovascular disease, systolic blood pressure, serum total cholesterol level, and first available eGFR

for designing and executing clinical trials, this finding is considered significant.

Although the main objective of this study was to evaluate the effects of change in eGFR on prognosis, a relation between an increase in change in eGFR over 1 year and increase in ESRD risk was observed. In the meta-analysis of the NKF-FDA Working Group, the relation between an increase in eGFR and risk of death was observed, but no association was seen for ESRD risk [8]. Past studies have, however, reported this association between increase in eGFR and ESRD risk [27, 28]. Several mechanisms have been proposed for the reasons why the increase in eGFR increases ESRD risk. For example, patients recovering from acute kidney injury may have shown increased eGFR. An increase in eGFR (decrease in serum creatinine level) may suggest loss of muscle mass or volume overload [8, 29]. However, this increase in eGFR over 2 years was not necessarily associated with an increase in ESRD risk in this study, thus the

association between an increase in eGFR and ESRD risk is not fully clarified at this stage.

The results of this study may extend beyond Japanese CKD patients, and it may be possible to generalize our findings to other East Asian countries or regions according to relatively similar lifestyles (high sodium intake, etc.) and risks of ESRD and cardiovascular events [30, 31]. In addition, because the meta-analyses of the NKF-FDA Working Group coincided with the results of the relation between change in eGFR and risk of ESRD in our study, it was suggested that universal surrogate endpoints can be used in joint international trials.

There are several limitations in this study. First, due to the comparatively short follow-up time, this disabled the evaluation of changes in eGFR over 3 years. In this study, the relative risk of changes in eGFR over 2 years was weaker than that over 1 year, and this may be due to the short follow-up. Second, given that there was no information on events after onset of ESRD, we could not evaluate the relation between changes 
Fig. 6 Relationships between percentage changes in eGFR and risk of ESRD by causes of nephropathy. Percentage change in eGFR over 1 year. a Diabetic nephropathy; b hypertensive nephropathy; c glomerular disease; $\mathbf{d}$ others. Percentage change in eGFR over 2 years. e Diabetic nephropathy; f hypertensive nephropathy; $\mathbf{g}$ glomerular disease; $\mathbf{h}$ others. The hazard ratio of ESRD according to percentage change in eGFR was adjusted for age, gender, DM, history of cardiovascular disease, systolic blood pressure, serum total cholesterol level, and first available eGFR a

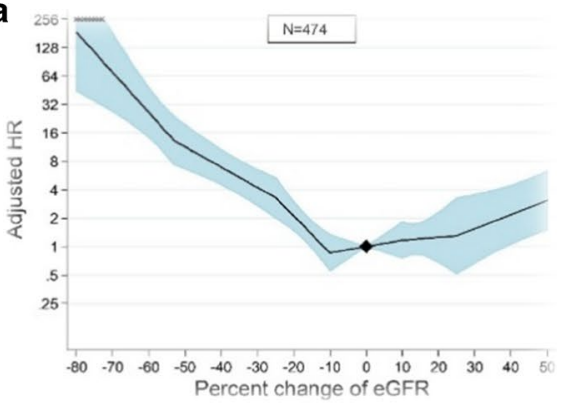

C

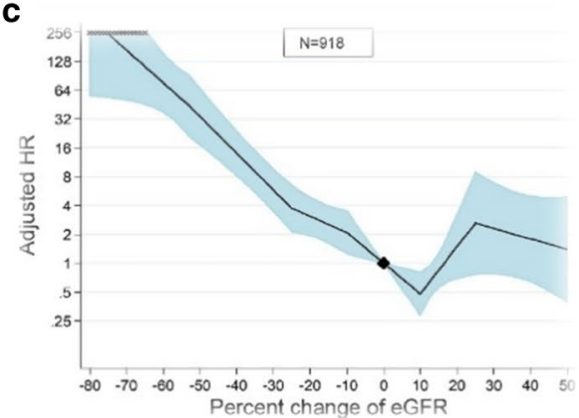

e

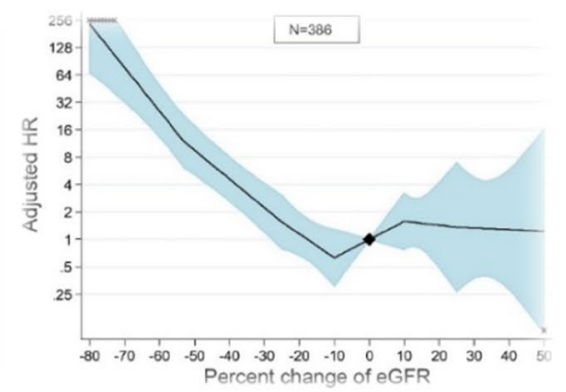

g

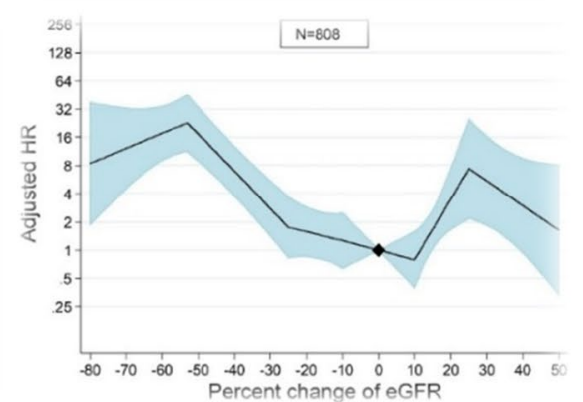

b

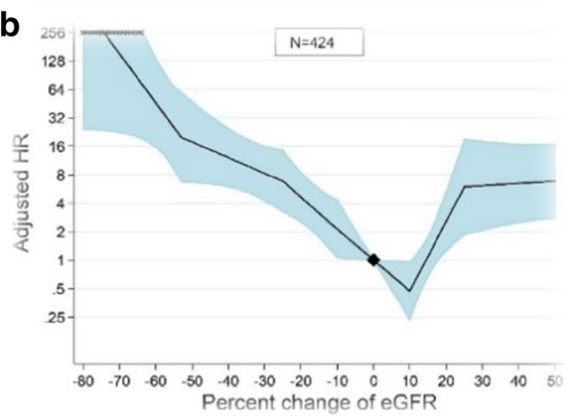

d

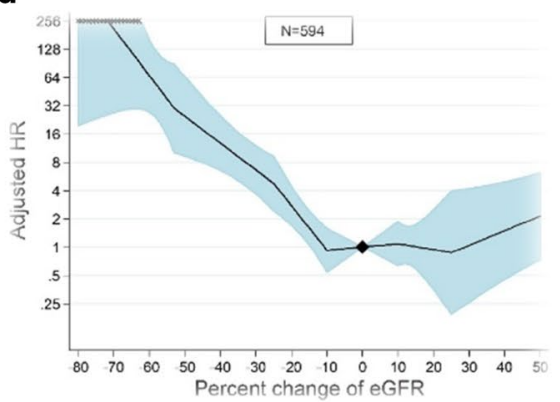

f

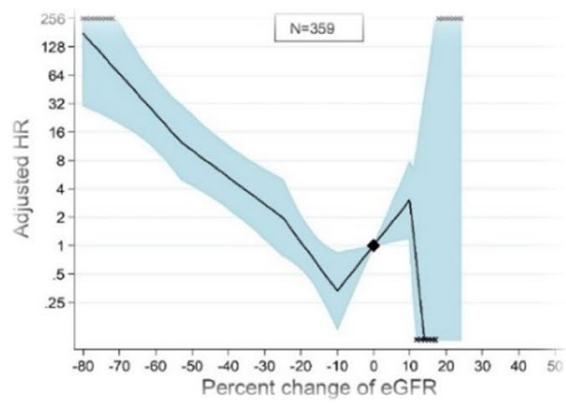

h

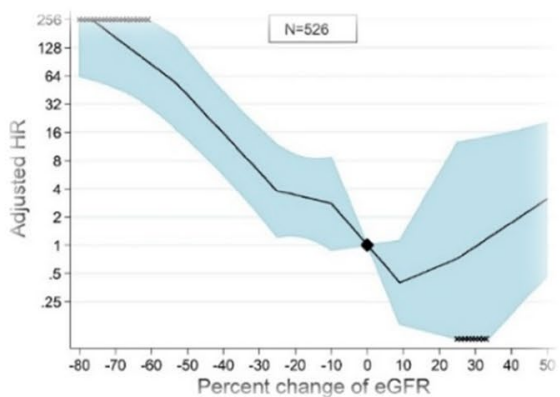

in eGFR and mortality. However, since there were records on deaths prior to ESRD, it was possible to review competing risks due to all deaths. Finally, although the possibility of threats of external validity of this study is low, we think that it is important to verify the relation between the decrease in eGFR and ESRD risk in other Asian countries and regions.

\section{Conclusion}

Association was confirmed between changes in eGFR less than the doubling of serum creatinine level and the risk of ESRD in Japanese CKD patients. This association was consistent in subgroups by causes of CKD including diabetic 


\begin{tabular}{|c|c|c|c|c|c|c|c|c|c|c|}
\hline \multirow{2}{*}{$\begin{array}{l}\text { Baseline eGFR1 } \\
\text { During 1-Year } \\
\text { Baseline Period }\end{array}$} & \multirow{2}{*}{$\begin{array}{l}\text { Follow-up After } \\
\text { Last eGFR1, y }\end{array}$} & \multicolumn{9}{|c|}{ Change in eGFR1 During 1-Year Baseline Period, \% } \\
\hline & & -53 & -40 & -30 & -25 & -20 & -10 & 0 & 10 & 25 \\
\hline \multirow{3}{*}{20} & 1 & $61(45,74)$ & $37(28,45)$ & $23(18,30)$ & $18(14,24)$ & $13(10,17)$ & $6.8(5.2,9.4)$ & $5(3.7,6.7)$ & $3.7(2.2,6.2)$ & $9.6(5.6,19)$ \\
\hline & 2 & $90(79,96)$ & $67(57,78)$ & $47(39,58)$ & $38(30,49)$ & $29(23,36)$ & $16(13,21)$ & $12(8.9,15)$ & $8.8(5.2,13)$ & $22(14,39)$ \\
\hline & 3 & $98(94,100)$ & $87(79,94)$ & $69(58,79)$ & $58(48,72)$ & $46(39,56)$ & $27(22,34)$ & $20(16,25)$ & $15(9.8,24)$ & $36(23,58)$ \\
\hline \multirow{3}{*}{25} & 1 & $36(24,49)$ & $19(14,26)$ & $12(8.7,16)$ & $8.9(6.6,13)$ & $6.4(4.9,8.7)$ & $3.2(2.4,4.3)$ & $2.4(1.7,3.1)$ & $1.8(1,2.9)$ & $4.7(2.7,9.2)$ \\
\hline & 2 & $66(49,81)$ & $41(31,51)$ & $26(20,35)$ & $20(15,28)$ & $15(11,19)$ & $7.8(6,10)$ & $5.7(4,7.4)$ & $4.2(2.5,6.7)$ & $11(6.8,21)$ \\
\hline & 3 & $86(71,95)$ & $61(49,74)$ & $42(33,56)$ & $34(26,47)$ & $25(20,34)$ & $14(10,18)$ & $10(7.3,13)$ & $7.5(4.5,12)$ & $19(12,33)$ \\
\hline \multirow{3}{*}{30} & 1 & $19(11,27)$ & $9.6(6.4,14)$ & $5.6(3.9,8.3)$ & $4.3(3,6.4)$ & $3.1(2.2,4.6)$ & $1.5(1.1,2.6)$ & $1.1(.76,1.6)$ & $.83(.46,1.5)$ & $2.2(1.2,4.2)$ \\
\hline & 2 & $40(25,55)$ & $22(15,30)$ & $13(9.4,18)$ & $10(7.1,15)$ & $7.3(5.3,9.9)$ & $3.7(2.7,5.2)$ & $2.7(1.8,3.6)$ & $2(1.2,3.4)$ & $5.3(3.1,10)$ \\
\hline & 3 & $60(41,77)$ & $36(24,48)$ & $23(16,32)$ & $18(13,26)$ & $13(9.4,18)$ & $6.7(4.9,9.2)$ & $4.9(3.4,6.7)$ & $3.6(2.1,6.2)$ & $9.5(5.6,17)$ \\
\hline \multirow{3}{*}{35} & 1 & $9.4(4.9,15)$ & $4.7(2.8,7.2)$ & $2.7(1.8,4.2)$ & $2(1.3,3.3)$ & $1.5(1,2.4)$ & $.73(.49,1.3)$ & $.54(.32, .79)$ & $.39(.2, .75)$ & $1.1(.56,2.2)$ \\
\hline & 2 & $21(12,33)$ & $11(6.6,17)$ & $6.5(4.2,9.8)$ & $4.9(3.2,7.4)$ & $3.5(2.4,5.6)$ & $1.8(1.2,3)$ & $1.3(.8,1.8)$ & $.96(.51,1.7)$ & $2.6(1.5,5.1)$ \\
\hline & 3 & $35(21,52)$ & $19(11,28)$ & $11(7.6,18)$ & $8.8(5.7,14)$ & $6.3(4.4,9.2)$ & $3.2(2.2,4.8)$ & $2.4(1.4,3.5)$ & $1.7(.94,3)$ & $4.6(2.5,8.4)$ \\
\hline \multirow{3}{*}{40} & 1 & $4.5(2.3,8.1)$ & $2.2(1.2,3.8)$ & $1.3(.78,2.4)$ & $.97(.6,1.9)$ & $.69(.44,1.2)$ & $.35(.22, .67)$ & $.25(.14, .42)$ & $.18(.09, .38)$ & $.5(.26,1.1)$ \\
\hline & 2 & $11(5.4,18)$ & $5.3(3,8.7)$ & $3.1(1.9,4.9)$ & $2.4(1.4,3.8)$ & $1.7(1.1,2.9)$ & $.84(.54,1.5)$ & $.62(.34, .94)$ & $.45(.22, .82)$ & $1.2(.63,2.4)$ \\
\hline & 3 & $19(10,30)$ & $9.5(5,15)$ & $5.6(3.3,8.8)$ & $4.2(2.5,7.1)$ & $3(1.9,4.7)$ & $1.5(.97,2.6)$ & $1.1(.63,1.8)$ & $.82(.4,1.5)$ & $2.2(1.2,4.1)$ \\
\hline \multirow{3}{*}{45} & 1 & $2.2(1,4.2)$ & $1.1(.55,2)$ & $.61(.34,1.2)$ & $.46(.26, .96)$ & $.33(.2, .6)$ & $.16(.1, .34)$ & $.12(.061, .22)$ & $.087(.038, .17)$ & $.24(.11, .5)$ \\
\hline & 2 & $5.2(2.4,9.6)$ & $2.6(1.3,4.5)$ & $1.5(.82,2.6)$ & $1.1(.64,2.3)$ & $.79(.48,1.4)$ & $.4(.24, .78)$ & $.29(.15, .48)$ & $.21(.093, .41)$ & $.57(.29,1.2)$ \\
\hline & 3 & $9.2(4.2,16)$ & $4.6(2.3,7.8)$ & $2.7(1.5,4.5)$ & $2(1.1,3.6)$ & $1.4(.84,2.6)$ & $.72(.43,1.4)$ & $.53(.29, .92)$ & $.39(.17, .76)$ & $1(.52,2)$ \\
\hline \multirow{3}{*}{50} & 1 & $1(.41,2.2)$ & $.5(.24,1)$ & $.29(.15, .61)$ & $.22(.12, .49)$ & $.15(.084, .3)$ & $.077(.043, .17)$ & $.056(.027, .11)$ & $.041(.016, .087)$ & $.11(.05, .24)$ \\
\hline & 2 & $2.5(1.1,5.2)$ & $1.2(.58,2.4)$ & $.7(.36,1.3)$ & $.53(.27,1)$ & $.37(.21, .73)$ & $.19(.1, .4)$ & $.14(.065, .25)$ & $.1(.041, .2)$ & $.27(.13, .6)$ \\
\hline & 3 & $4.5(1.8,8.6)$ & $2.2(1,4.1)$ & $1.3(.67,2.3)$ & $.96(.5,1.9)$ & $.68(.38,1.3)$ & $.34(.19, .72)$ & $.25(.13, .47)$ & $.18(.075, .37)$ & $.49(.24,1.1)$ \\
\hline
\end{tabular}

Color scale based on predicted risks

$\begin{array}{llllllllllll}100 & 80 & 60 & 40 & 20 & 15 & 10 & 5 & 3.75 & 2.5 & 1 & 0.5\end{array}$

Fig. 7 Predicted risk of ESRD and change in eGFR over 1 year. Values are estimated 1- to 3-year risk of ESRD (\%) with 95\% confidence interval. Other covariates were set as follows: age, 60 years; gender, male; systolic blood pressure, $130 \mathrm{~mm} \mathrm{Hg}$; total cholesterol level, $5 \mathrm{mmol} / \mathrm{l}$; and no history of DM or cardiovascular disease. Competing risk model was examined taking all-cause mortality as the competing endpoint. Serum total cholesterol level of $1 \mathrm{mmol} / \mathrm{l}$ is $38.67 \mathrm{mg} / \mathrm{dL}$

\begin{tabular}{|c|c|c|c|c|c|c|c|c|c|c|}
\hline \multirow{2}{*}{$\begin{array}{l}\text { Baseline eGFR1 } \\
\text { During 2-Year } \\
\text { Baseline Period }\end{array}$} & \multirow{2}{*}{$\begin{array}{l}\text { Follow-up After } \\
\text { Last eGFR1, y }\end{array}$} & \multicolumn{9}{|c|}{ Change in eGFR1 During 2-Year Baseline Period, \% } \\
\hline & & -53 & -40 & -30 & -25 & -20 & -10 & 0 & 10 & 25 \\
\hline & 1 & $66(53,76)$ & $35(30,43)$ & $19(15,24)$ & $14(11,18)$ & $11(8.9,14)$ & $6.4(4,9.6)$ & $6.7(4.7,9.2)$ & $6.9(3.5,11)$ & $9.2(3.4,20)$ \\
\hline \multirow[t]{3}{*}{20} & 2 & $91(81,97)$ & $63(52,72)$ & $39(31,49)$ & $29(23,39)$ & $23(18,29)$ & $14(9,20)$ & $15(9.9,19)$ & $15(7.5,23)$ & $20(7.1,36)$ \\
\hline & 3 & $95(88,99)$ & $70(60,81)$ & $45(36,58)$ & $34(27,48)$ & $27(22,36)$ & $17(11,24)$ & $17(12,23)$ & $18(8.7,27)$ & $23(8,42)$ \\
\hline & 1 & $41(32,53)$ & $19(16,24)$ & $10(8,13)$ & $7.3(5.5,9.6)$ & $5.6(4.6,7.3)$ & $3.2(2.1,4.8)$ & $3.4(2.4,4.9)$ & $3.5(1.6,5.9)$ & $4.7(1.4,10)$ \\
\hline \multirow[t]{3}{*}{25} & 2 & $70(57,83)$ & $39(31,49)$ & $22(17,28)$ & $16(12,21)$ & $12(9.8,16)$ & $7.2(4.9,10)$ & $7.5(5.3,10)$ & $7.7(3.7,12)$ & $10(3.5,19)$ \\
\hline & 3 & $77(64,88)$ & $45(36,57)$ & $26(20,34)$ & $19(14,26)$ & $15(11,20)$ & $8.7(5.8,13)$ & $9(6.6,12)$ & $9.3(4.5,14)$ & $12(4.1,24)$ \\
\hline & 1 & $23(17,33)$ & $10(7.8,14)$ & $5.2(3.8,7.3)$ & $3.7(2.7,5.2)$ & $2.8(2.2,4)$ & $1.6(1.1,2.5)$ & $1.7(1.2,2.5)$ & $1.7(.94,3.1)$ & $2.4(.75,5.1)$ \\
\hline \multirow[t]{3}{*}{30} & 2 & $45(33,61)$ & $22(16,30)$ & $11(8.2,15)$ & $8.2(5.8,11)$ & $6.3(4.9,8.6)$ & $3.6(2.5,5.4)$ & $3.8(2.9,5.5)$ & $3.9(2.1,6.2)$ & $5.3(1.8,10)$ \\
\hline & 3 & $52(39,67)$ & $25(19,34)$ & $14(9.6,19)$ & $9.8(6.8,14)$ & $7.5(5.5,11)$ & $4.4(3,6.7)$ & $4.6(3.5,6.5)$ & $4.7(2.5,7.5)$ & $6.4(2,12)$ \\
\hline & 1 & $12(8.2,19)$ & $5.2(3.8,8)$ & $2.6(1.9,4)$ & $1.8(1.3,2.9)$ & $1.4(1,2.2)$ & $.81(.52,1.3)$ & $.84(.59,1.3)$ & $.87(.48,1.6)$ & $1.2(.37,2.6)$ \\
\hline \multirow[t]{3}{*}{35} & 2 & $26(17,40)$ & $11(7.7,17)$ & $5.8(4.1,8.7)$ & $4.1(2.8,6.2)$ & $3.2(2.4,4.7)$ & $1.8(1.2,2.9)$ & $1.9(1.4,2.9)$ & $2(1.1,3.3)$ & $2.6(.88,5.4)$ \\
\hline & 3 & $30(20,45)$ & $14(9.2,21)$ & $7(4.7,11)$ & $5(3.2,7.5)$ & $3.8(2.6,5.6)$ & $2.2(1.5,3.6)$ & $2.3(1.8,3.7)$ & $2.4(1.3,4.1)$ & $3.2(1,6.3)$ \\
\hline & 1 & $6.3(3.8,11)$ & $2.6(1.8,4.4)$ & $1.3(.9,2.2)$ & $.92(.61,1.6)$ & $.7(.49,1.1)$ & $.4(.25, .69)$ & $.42(.28, .69)$ & $.43(.25, .86)$ & $.58(.18,1.3)$ \\
\hline \multirow[t]{3}{*}{40} & 2 & $14(8.5,24)$ & $5.8(3.8,9.9)$ & $2.9(1.9,4.8)$ & $2.1(1.3,3.3)$ & $1.6(1.1,2.5)$ & $.91(.58,1.5)$ & $.94(.65,1.6)$ & $.98(.55,1.8)$ & $1.3(.44,2.9)$ \\
\hline & 3 & $16(9.7,28)$ & $7(4.6,13)$ & $3.5(2.3,6.2)$ & $2.5(1.5,4.3)$ & $1.9(1.3,3.1)$ & $1.1(.69,1.9)$ & $1.1(.82,2)$ & $1.2(.68,2.2)$ & $1.6(.5,3.4)$ \\
\hline & 1 & $3.2(1.7,5.8)$ & $1.3(.82,2.4)$ & $.64(.42,1.2)$ & $.46(.29, .86)$ & $.35(.23, .66)$ & $.2(.12, .37)$ & $.21(.13, .36)$ & $.21(.12, .45)$ & $.29(.083, .66)$ \\
\hline \multirow[t]{3}{*}{45} & 2 & $7(4,14)$ & $2.9(1.8,5.5)$ & $1.5(.91,2.7)$ & $1(.63,1.8)$ & $.78(.5,1.4)$ & $.45(.28, .78)$ & $.47(.31, .83)$ & $.48(.26, .96)$ & $.66(.22,1.5)$ \\
\hline & 3 & $8.5(4.5,16)$ & $3.5(2.1,6.7)$ & $1.8(1.1,3.3)$ & $1.3(.74,2.3)$ & $.95(.59,1.7)$ & $.55(.34,1)$ & $.57(.37, .99)$ & $.59(.33,1.2)$ & $.8(.25,1.8)$ \\
\hline & 1 & $1.6(.78,3.5)$ & $.64(.37,1.3)$ & $.32(.2, .65)$ & $.23(.14, .62)$ & $.17(.11, .35)$ & $.099(.054, .2)$ & $.1(.059, .19)$ & $.11(.05, .24)$ & $.14(.042, .35)$ \\
\hline \multirow[t]{2}{*}{50} & 2 & $3.6(1.8,7.9)$ & $1.5(.84,3)$ & $.73(.43,1.5)$ & $.51(.3,1)$ & $.39(.22, .78)$ & $.22(.13, .41)$ & $.23(.14, .44)$ & $.24(.13, .51)$ & $.33(.11, .84)$ \\
\hline & 3 & $4.3(2.1,9)$ & $1.8(.98,3.7)$ & $.88(.5,1.8)$ & $.62(.35,1.2)$ & $.47(.27, .94)$ & $.27(.16, .53)$ & $.28(.17, .52)$ & $.29(.15, .66)$ & $.4(.13,1.1)$ \\
\hline
\end{tabular}

\section{Color scale based on predicted risks}

$\begin{array}{llllllllllll}100 & 80 & 60 & 40 & 20 & 15 & 10 & 5 & 3.75 & 2.5 & 0.5\end{array}$

Fig. 8 Predicted risk of ESRD and change in eGFR over 2 years. Values are estimated 1- to 3-year risk of ESRD (\%) with 95\% confidence interval. Other covariates were set as follows: age, 60 years; gender, male; systolic blood pressure, $130 \mathrm{~mm} \mathrm{Hg}$; total cholesterol level, $5 \mathrm{mmol} / \mathrm{l}$; and no history of DM or cardiovascular disease. Competing risk model was examined taking all-cause mortality as the competing endpoint. Serum total cholesterol level of $1 \mathrm{mmol} / \mathrm{l}$ is $38.67 \mathrm{mg} / \mathrm{dL}$ 
nephropathy and age. The NKF-FDA Working Group recommends the use of more than $40 \%$ decrease in eGFR as a surrogate endpoint to various situations and more than $30 \%$ decrease to limited situations (e.g., no acute effects of drugs). The results of this study indicate that this approach is reasonable even in Japanese CKD patients.

\section{Assessment of percentage change in eGFR as surrogate endpoint for end-stage renal disease: analysis of data of general health examinations in Okinawa prefecture and ORIENT study}

\section{Epidemiological study}

\section{Method}

Data The data of 69,727 subjects who were screened in health examinations by the Okinawa General Health Maintenance Association (OGHMA) from 1993 to 1996 were used in this study [32]. Of this, the data of 69,238 subjects without missing values in the baseline data at the start of the study were analyzed. Serum creatinine levels at the last eGFR at the baseline period were not imputed. Taking the development of ESRD (initiation of dialysis) to be the endpoint, 15-year follow-up survey was conducted. No data on deaths were obtained. As serum creatinine levels were measured using the Jaffe reaction, they were converted so that they conformed to enzymatic method levels [32]. eGFR was calculated using the equation developed by Japanese Society of Nephrology [23]:

$\operatorname{eGFR}\left(\mathrm{ml} / \mathrm{min} / 1.73 \mathrm{~m}^{2}\right)=194 \times \mathrm{Cr}^{-1.094} \times \mathrm{age}^{-0.287}$, note: 0.739 times in the case of female. $\mathrm{Cr}$ is serum creatinine level $(\mathrm{mg} / \mathrm{dl})$.

Percentage changes in eGFR were calculated using the following equation [8]:

Percentage changes in eGFR (\%/observational period)

$=\frac{\text { (last eGFR at baseline period }- \text { first available eGFR) }}{\text { first available eGFR }} \times 100$.

note: First available eGFR and last eGFR at baseline period indicate the first available eGFR and last eGFR. The observational period was $1-3$ years.
Statistical analyses Subjects with eGFR $60 \mathrm{ml} / \mathrm{min} / 1.73 \mathrm{~m}^{2}$ or more at the start of the study were defined as the higheGFR group and those with eGFR $60 \mathrm{ml} / \mathrm{min} / 1.73 \mathrm{~m}^{2}$ or less as the low-eGFR group. Percentage change in eGFR every $10 \%$ was set as the cutoff level [8]. The relationship between the percentage change in eGFR and the time to ESRD onset was evaluated using the Cox proportional hazards model. With this model, percentage changes in eGFR were used as continuous variables, and adjustments were made with baseline characteristics such as gender, age, body mass index (BMI), systolic blood pressure, serum total cholesterol level, eGFR, and urinary protein level (dipstick). aHRs and 95\% CIs were acquired using spline curves. Next, the positive predictive value (PPV) of ESRD onset for each cutoff level was calculated taking sensitivity and specificity to be fixed values, and assuming prevalence to vary:

PPV (\%)

$=\frac{\text { sensitivity } \times \text { prevalence } \times 100}{\text { sensitivity } \times \text { prevalence }+(1-\text { prevalence }) \times(1-\text { specificity })}$.

Details of the study results have been reported in "Clinical and Experimental Nephrology". An outline is provided below [33].

\section{Results}

The characteristics of each group are summarized in Table 4 . The distributions of percentage changes in eGFR are shown in Fig. 9. The high-eGFR group consisted of 58,292 subjects, and data on percentage changes in eGFR over 1-3 years were obtained from 47,688 subjects, 43,381 subjects, and 42,061 subjects, respectively. The low-eGFR group consisted of 10,946 subjects, and data on percentage changes in eGFR over 1-3 years were obtained from 8991 subjects, 8017 subjects, and 7787 subjects, respectively. Subjects with decrease in percentage change in eGFR of more than $40 \%$ over $1-3$ years were 319 subjects $(0.67 \%)$, 338 subjects $(0.78 \%)$, and 601 subjects $(1.43 \%)$, respectively, in the high-eGFR group, and 70 subjects $(0.78 \%), 59$ subjects $(0.74 \%)$, and 55 subjects $(0.71 \%)$, respectively, in the low-eGFR group.

The relationships between percentage change in eGFR and risk of ESRD were evaluated. In the high-eGFR group, the risk of ESRD tended to be high with decreases in percentage change in eGFR of more than $30 \%$ over 2 or 3 years 
Table 4 Baseline characteristics

\begin{tabular}{llll}
\hline & All & High-eGFR group & Low-eGFR group \\
\hline $\mathrm{N}(\%)$ & 69,238 & $58,292(84.2)$ & $10,946(15.8)$ \\
Male $(\%)$ & $29,744(43.0)$ & $25,958(44.5)$ & $3786(34.6)$ \\
Age $($ years $)$ & $55.6 \pm 14.7$ & $53.19 \pm 14.2$ & $68.39 \pm 10.2$ \\
BMI $\left(\mathrm{kg} / \mathrm{m}^{2}\right)$ & $24.1 \pm 3.4$ & $24.05 \pm 3.4$ & $24.27 \pm 3.3$ \\
$\mathrm{SBP}(\mathrm{mmHg})$ & $127.8 \pm 17.4$ & $126.68 \pm 17.2$ & $133.77 \pm 17.4$ \\
Total cholesterol level $(\mathrm{mg} / \mathrm{dl})$ & $204.3 \pm 35.6$ & $203.1 \pm 35.5$ & $210.7 \pm 35.9$ \\
Urinary protein $(\%)$ & & & \\
- & $66,737(96.4)$ & $56,543(97)$ & $10,194(93.1)$ \\
\pm & $1671(2.4)$ & $1224(2.1)$ & $447(4.1)$ \\
$1+$ & $830(1.2)$ & $525(0.9)$ & $305(2.8)$ \\
eGFR (ml/min/1.73 $\left.\mathrm{m}^{2}\right)$ & $80.2 \pm 21.2$ & $85.4 \pm 18.7$ & $52.4 \pm 7.0$ \\
Percentage change in eGFR over 1 year $(\% / y e a r)$ & $1.0 \pm 19.4$ & $0.8 \pm 19.5$ & $2.0 \pm 19.0$ \\
Percentage change in eGFR over 2 years $(\% / 2$ years) & $0.5 \pm 20.9$ & $0.1 \pm 19.5$ & $2.6 \pm 27.0$ \\
Percentage change in eGFR over 3 years (\%/3 years) & $-0.3 \pm 21.3$ & $-2.4 \pm 20.2$ & $10.7 \pm 23.5$ \\
ESRD risk (\%) & $383(0.55)$ & $186(0.32)$ & $197(1.8)$ \\
\hline
\end{tabular}

Mean \pm standard deviation and number of cases (\%) are shown

$B M I$ body mass index, $S B P$ systolic blood pressure, $e G F R$ estimated glomerular filtration rate, ESRD endstage renal disease

A

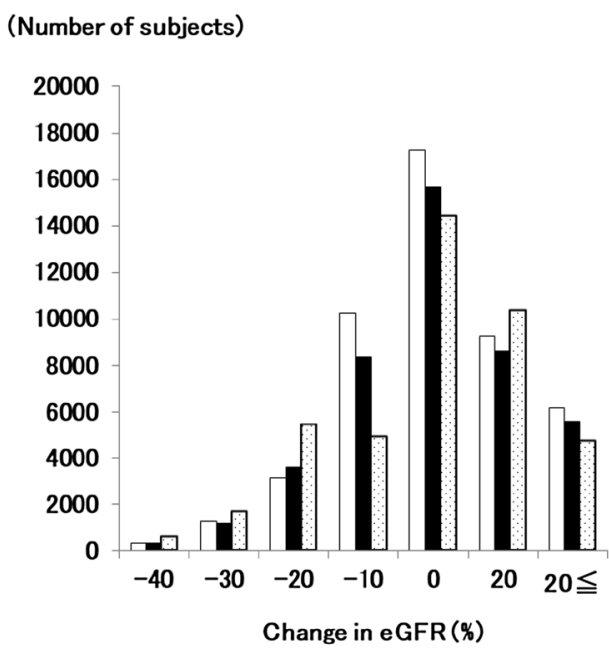

Fig. 9 Distribution of percentage changes in eGFR. Percentage changes in eGFR and frequency at each baseline period are shown. Percentage changes in eGFR of less than $40 \%$ are indicated as $-40 \%$. The same applies in other figures below. a High-eGFR group: percentage change in eGFR over 1 year: 47,688 subjects, percentage

(Fig. 10). In the low-eGFR group, the risk of ESRD also tended to be high with decreases in percentage change in eGFR of more than $20 \%$ over 2 years and of more than $30 \%$ over 3 years (Fig. 11). Both the high- and low-eGFR groups did not show any correlation between the decrease in percentage change in eGFR over 1 year and increased risk of $\square 1$ year

- 2 years $\square 3$ years

B

(Number of subjects)

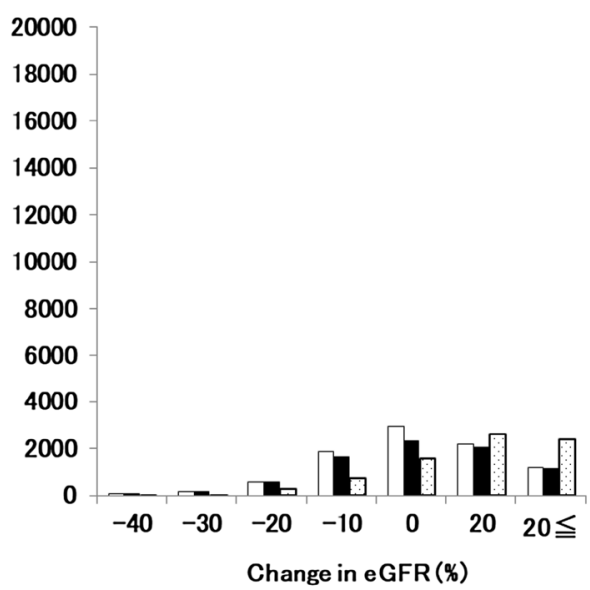

change in eGFR over 2 years: 43,381 subjects, percentage change in eGFR over 3 years: 42,061 subjects. b Low-eGFR group: percentage change in eGFR over 1 year: 8991 subjects, percentage change in eGFR over 2 years: 8017 subjects, percentage change in eGFR over 3 years: 7787 subjects. $e G F R$ estimated glomerular filtration rate

ESRD. PPV was the highest at the percentage change in eGFR of $30 \%$ in the high-eGFR group, and high at the percentage changes in eGFR of $30 \%$ and $40 \%$ in the low-eGFR group (Fig. 12). 
Fig. 10 Relationships between percentage changes in eGFR and risk of ESRD in higheGFR group. aHR adjusted for baseline characteristics (gender, age, BMI, systolic blood pressure, serum total cholesterol level, eGFR, and protein level) and 95\% CI are shown. a Percentage change in eGFR over 1 year; $-40 \%$ aHR 0.70 (95\% CI, 0.32-1.54), $-30 \% 0.92(0.55-1.56),-20 \%$ 1.22 (0.79-1.88). b Percentage change in eGFR over 2 years; $-40 \% 1.43(0.79-2.57),-30 \%$ 1.38 (0.84-2.28), - $20 \% 1.32$ (0.82-2.14). c Percentage change in eGFR over 3 years; $-40 \% 1.74$ (1.01-2.99), - 30\% $1.49(0.91-2.43),-20 \% 1.27$ (0.80-2.01). ESRD end-stage renal disease, $B M I$ body mass index, $a H R$ adjusted hazard ratio, $C I$ confidence interval
A aHR

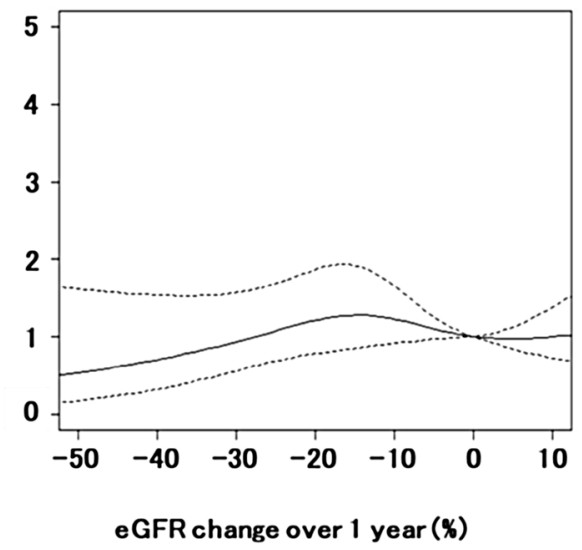

C

aHR

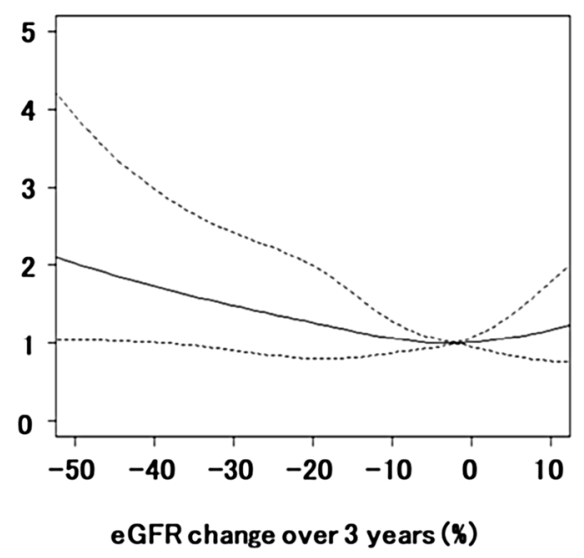

B

aHR

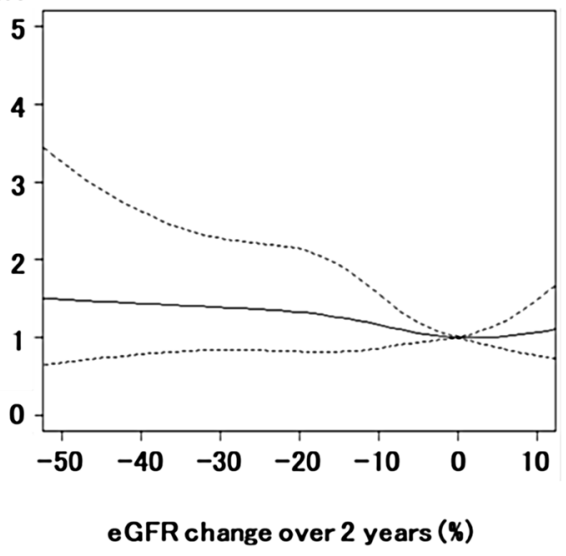

\section{Clinical study}

\section{Method}

Data In the Olmesartan Reducing Incidence of End-Stage Renal Disease in Diabetic kidney disease Trial (ORIENT) study, 566 patients with type 2 diabetes accompanied by overt nephropathy were followed up for 5 years, to evaluate the effects of olmesartan for reducing the progression of nephropathy [34]. These data were analyzed in this study.

Analysis The onset of ESRD (initiation of dialysis) during the entire study period was defined as the true endpoint. The percentage changes in eGFR for a baseline period over 1-3 years were obtained in the same way using the above method. Percentage change in eGFR every $10 \%$ was set as the cutoff level. These were set as the surrogate endpoints. In the evaluation of the surrogate endpoints, the surrogate endpoints or true endpoints during the observational period were analyzed as time-to-event data. First, the relationship between the percentage changes in eGFR and time-to-true endpoint was evaluated using Cox proportional hazards models. With this Cox proportional hazard model, percentage changes in eGFR were used as continuous variables, then adjusted for the olmesartan usage and baseline characteristics [eGFR, urinary albumin-tocreatinine ratio (UACR), and country] referring to earlier papers. aHR and 95\% CI were obtained using spline curves [35]. Next, using the Cox proportional hazard model adjusted for the baseline characteristics, the aHRs for true endpoints and for surrogate endpoints in the olmesartan group were calculated, respectively. After this, the bootstrap method was used to calculate the aHR for true endpoints and surrogate endpoints in the Cox proportional hazard model adjusted for the baseline characteristics, and their ratios and 95\% CI were obtained [36]:

aHR ratio $=\frac{\text { aHR for true endpoint }}{\text { aHR for surrogate endpoint }}$.

The closer this aHR is to 1 , the more the aHR of the true endpoint and that of the surrogate endpoint match. 
Fig. 11 Relationships between percentage changes in eGFR and risk of ESRD in loweGFR group. aHR adjusted for baseline characteristics (gender, age, BMI, systolic blood pressure, serum total cholesterol level, eGFR, and urinary protein level) and $95 \%$ CI are shown. a Percentage change in eGFR over 1 year; - $40 \%$ aHR $0.74(0.44-1.25)$ $-30 \% 0.82(0.51-1.33),-20 \%$ 0.90 (0.54-1.48). b Percentage change in eGFR over 2 years; $-40 \% 1.57$ (0.94-2.62), - 30\% $1.49(0.92-2.43),-20 \% 1.41$ (0.87-2.30). c Percentage change in eGFR over 3 years; $-40 \% 1.68(1.17-2.40),-30 \%$ $1.38(1.09-1.76),-20 \% 1.12$ (1.00-1.25). ESRD end-stage renal disease, $B M I$ body mass index, $a H R$ adjusted hazard ratio, $C I$ confidence interval
A aHR

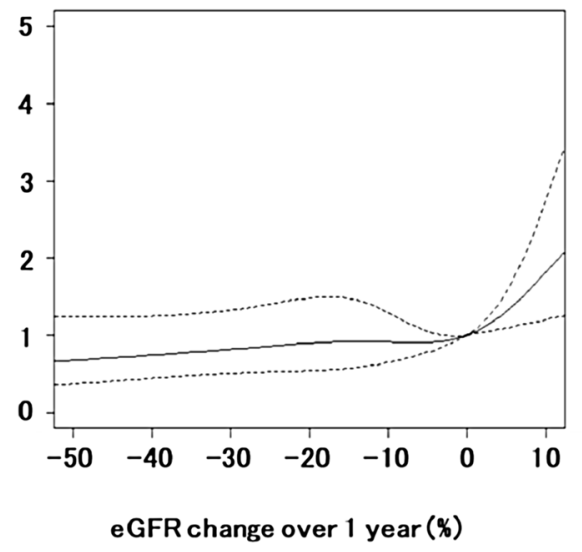

C aHR

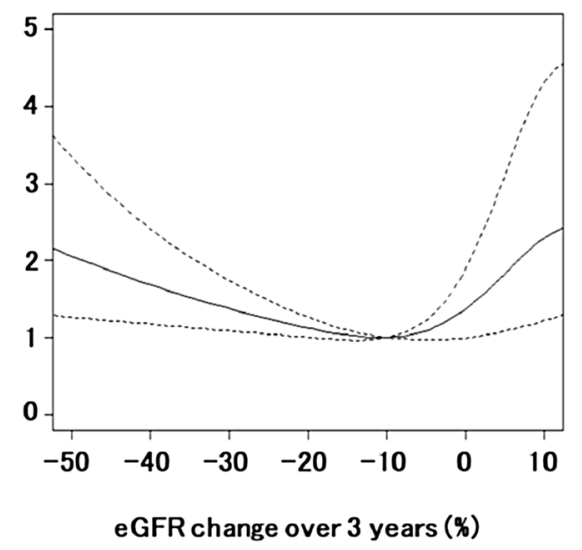

B aHR

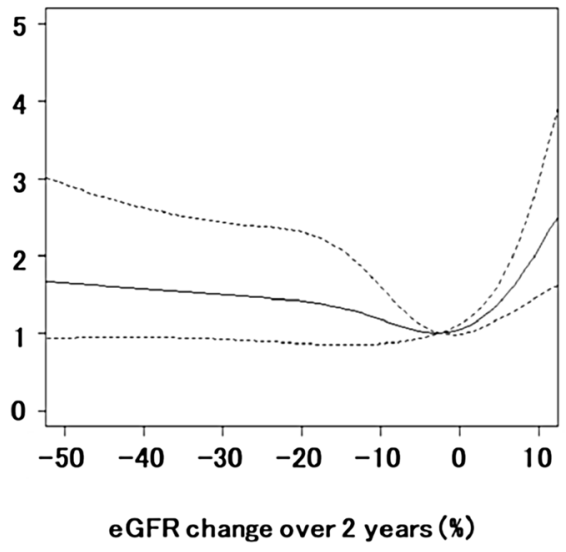

\section{Results}

The characteristics of each group are summarized in Table 5. The distributions of percentage changes in eGFR are shown in Fig. 13. The olmesartan group consisted of 282 subjects, and data on percentage changes in eGFR over 1-3 years were obtained from 248 subjects, 205 subjects, and 135 subjects, respectively. The control group consisted of 284 subjects, and data on percentage changes in eGFR over 1-3 years were obtained from 252 subjects, 193 subjects, and 126 subjects, respectively. Subjects with decrease in percentage changes in eGFR of more than $60 \%$ over $1-3$ years were 8 subjects (3.2\%), 16 subjects $(7.8 \%)$, and 14 subjects $(10.4 \%)$, respectively, in the olmesartan group, and 6 subjects (2.4\%), 13 subjects $(6.7 \%)$, and 16 subjects $(12.7 \%)$, respectively, in the control group. Subjects with decrease in percentage changes in eGFR of more than $50 \%$ over $1-3$ years were 17 subjects $(6.9 \%), 35$ subjects $(17.1 \%)$, and 29 subjects $(21.5 \%)$, respectively, in the olmesartan group, and 13 subjects (5.2\%), 32 subjects
(16.6\%), and 29 subjects (23.0\%), respectively, in the control group.

The relationships between percentage changes in eGFR and risk of ESRD were evaluated. Although the 95\% CI of aHR was wide and evaluation was restricted, the greater the decrease in percentage changes in eGFR over 1-2 years, the higher was the ESRD risk (Fig. 14). No evident correlation was found for the percentage changes in eGFR over 3 years.

As for the effects of olmesartan on surrogate endpoints, when the decrease in percentage changes in eGFR was more than $20 \%$ over 1-2 years, there was a tendency for aHR to be less than 1 like true endpoints (Fig. 15). However, it was more than 1 only when the percentage change in eGFR over 1 year was $40 \%$. Next, evaluation of the aHR of the true endpoint and surrogate endpoint indicated the aHR to be a value close to 1 when the percentage changes in eGFR over 2 years ranged from 20 to $40 \%$. The aHR was closest to 1 at $40 \%$ (Fig. 16). 
Fig. 12 Percentage changes in eGFR and positive predictive value of ESRD. a Percentage change in eGFR over 2 years in high-eGFR group. b Percentage change in eGFR over 3 years in high-eGFR group. c Percentage change in eGFR over 2 years in low-eGFR group. $\mathbf{d}$ Percentage change in eGFR over 3 years in low-eGFR group. $P P V$ positive predictive value
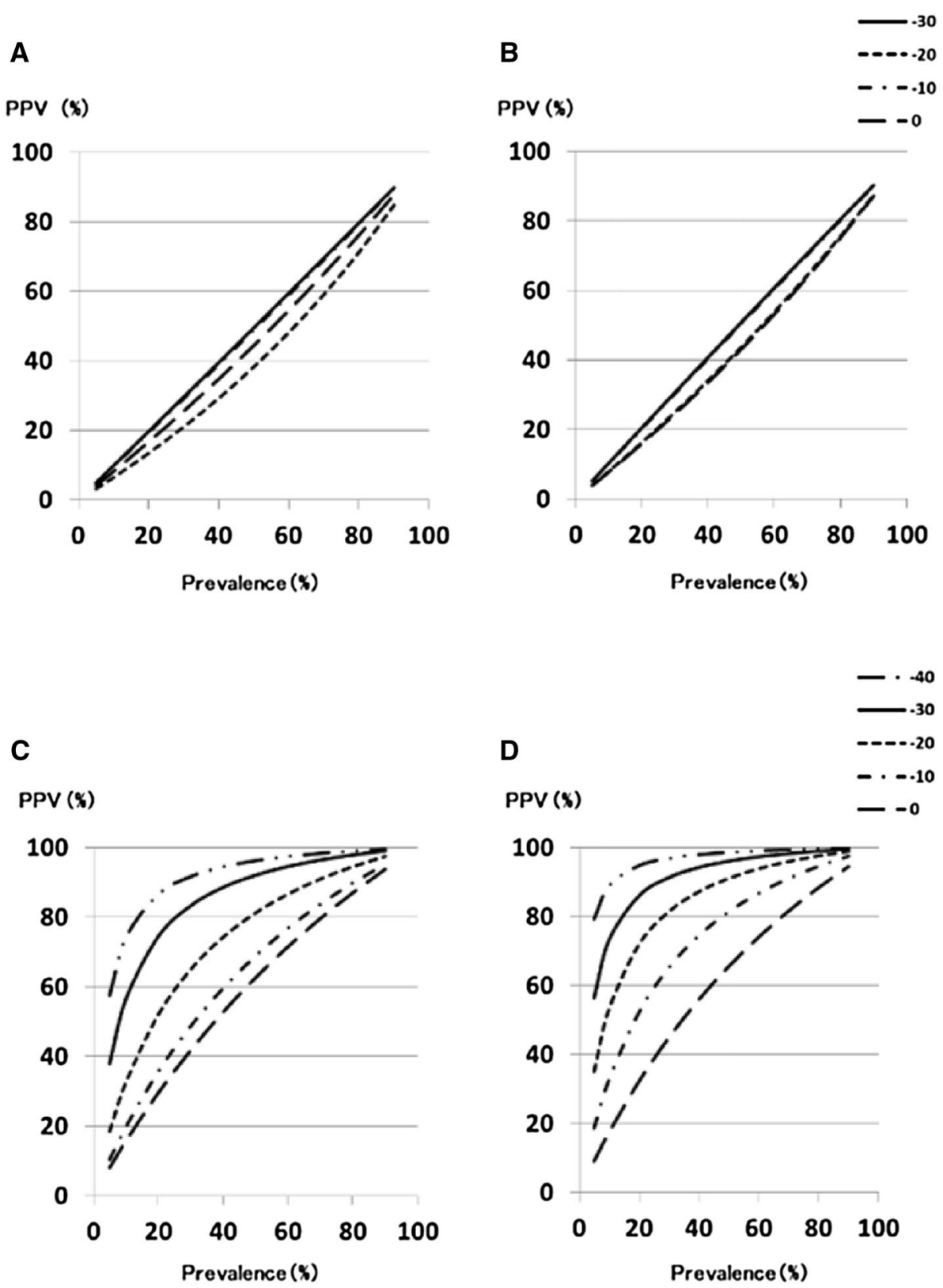

\section{Discussion}

In this study, the data of epidemiological and clinical studies were used to evaluate the relationships between true endpoints and surrogate endpoints. To date, epidemiological cohort studies on CKD patients in Japan have reported the correlation between percentage changes in eGFR and risk of ESRD [18,20]. According to the study on CKD patients by Chang et al., decrease in percentage changes in eGFR of more than $30 \%$ over 2 years correlated with ESRD strongly [18]. On the other hand, in a CKD Japan Cohort (Chronic Kidney Disease Japan Cohort, CKD-JAC) study, the relationship between patient distribution and risk of ESRD based on percentage changes in eGFR over 1-2 years indicated 30 or $40 \%$ to be the surrogate endpoints [18]. In this study, the differences in patient background by age, gender, and the causes of CKD were evaluated, and the same correlation between percentage changes in eGFR and risk of ESRD was seen for each factor [20]. In this study, to investigate subjects different from these studies, the data of health examinations conducted in Okinawa, Japan, were analyzed.

The relationships between percentage changes in eGFR as ESRD surrogate marker and risk of ESRD was investigated. Both the high-eGFR group and low-eGFR group had very few subjects showing decrease in percentage changes in eGFR of more than $40 \%$. In both groups, the greater the decrease in percentage changes in eGFR of more than $30 \%$ over 2-3 years, the higher was the risk of ESRD. 
Table 5 Baseline characteristics

\begin{tabular}{lll}
\hline & Olmesartan group & Control group \\
\hline Number of cases & 282 & 284 \\
Age (years) & $59.1 \pm 8.1$ & $59.2 \pm 8.1$ \\
Country (Japan/China) & $182 / 100$ & $184 / 100$ \\
Male $(\%)$ & $199(70.6)$ & $192(67.6)$ \\
Smoking $(\%)$ & $72(25.5)$ & $72(25.4)$ \\
BMI $\left(\mathrm{kg} / \mathrm{m}^{2}\right)$ & $25.3 \pm 4.2$ & $25.3 \pm 3.8$ \\
SBP $(\mathrm{mmHg})$ & $141.7 \pm 17.0$ & $140.8 \pm 18.0$ \\
DBP $(\mathrm{mmHg})$ & $77.8 \pm 10.4$ & $77.2 \pm 10.6$ \\
Albuminuria (mg/mmol) & $192.3(87.1,339.4)$ & $191.2(98.4,352.9)$ \\
HbA1c $(\%)$ & $7.1 \pm 1.2$ & $7.1 \pm 1.2$ \\
eGFR (ml/min/1.73 $\left.\mathrm{m}^{2}\right)$ & $37.1 \pm 9.6$ & $37.1 \pm 10.0$ \\
Percentage change in eGFR over 1 year $(\% /$ year) & $-19.2 \pm 18.2$ & $-18.4 \pm 17.0$ \\
Percentage change in eGFR over 2 years $(\% / 2$ years) & $-27.8 \pm 22.1$ & $-28.4 \pm 21.1$ \\
Percentage change in eGFR over 3 years (\%/3 years) & $-29.2 \pm 22.2$ & $-32.3 \pm 21.7$ \\
ESRD risk (\%) & $74(26.2)$ & $78(27.5)$ \\
\hline
\end{tabular}

Mean \pm standard deviation, number of cases (\%), median value (first quartile, third quartile) are shown $B M I$ body mass index, $S B P$ systolic blood pressure, $D B P$ diastolic blood pressure, $H b A 1 c$ hemoglobin A1c, $e G F R$ estimated glomerular filtration rate, ESRD end-stage renal disease

Fig. 13 Distribution of percentage changes in eGFR. Percentage changes in eGFR and frequency at each baseline period are shown. Olmesartan group: percentage changes in eGFR over 1 year: 248 subjects, percentage changes in eGFR over 2 years: 205 subjects, percentage changes in eGFR over 3 years: 135 subjects. Control group: percentage changes in eGFR over 1 year: 252 subjects, percentage changes in eGFR over 2 years: 193 subjects, percentage changes in eGFR over 3 years: 126 subjects

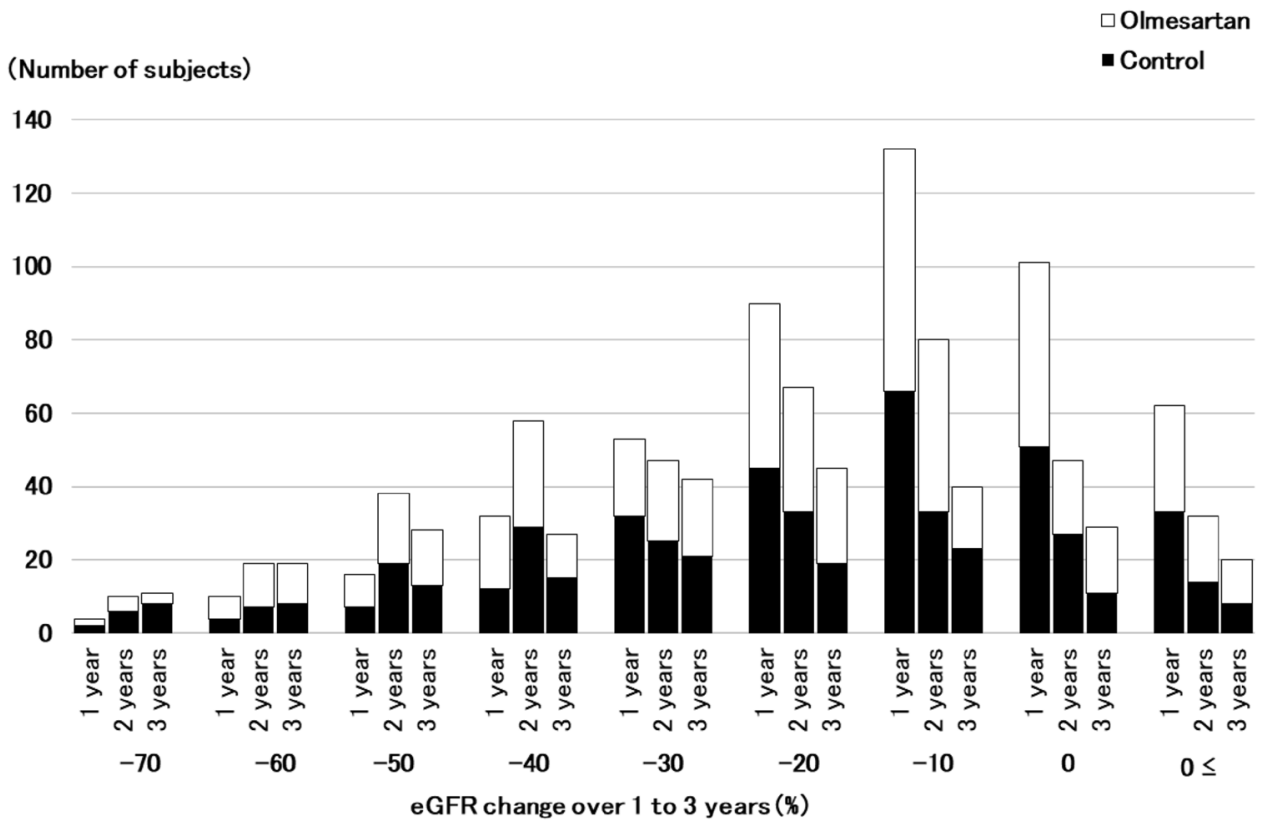

However, the percentage changes in eGFR over 1 year did not show such tendency. Coresh et al. reported correlation between percentage changes in eGFR over 1-3 years and risk of ESRD in both the high-eGFR group and low-eGFR group [8]. In this analysis, correlation between percentage changes in eGFR over 1 year and risk of ESRD could not be demonstrated easily because there were fewer subjects showing decrease in percentage changes in eGFR of more than $40 \%$ than the study by Coresh et al., which may be why there were only a few ESRD onsets.

Next, the cutoff levels for percentage changes in eGFR over 2 or 3 years were investigated. Studies on PPV showed that cutoff levels indicating high PPV were $30 \%$ in the high-eGFR group, and 30 or $40 \%$ in the loweGFR group. Therefore, based on these findings, percentage change in eGFR of 30 or $40 \%$ over 2 or 3 years was 
Fig. 14 Relationships between percentage changes in eGFR and risk of ESRD. Logarithm of aHR adjusted for baseline characteristics (use of olmesartan, eGFR, UACR, and country) and $95 \% \mathrm{CI}$ are shown. A Percentage change in eGFR over 1 year; $-50 \%$ aHR 26.77 (95\% CI 9.59-74.74), - 40\% 11.95 (4.33-32.97), $-30 \%$ 5.74 (2.05-16.07), - 20\% 3.02 (0.93-9.83). B Percentage change in eGFR over 2 years; $-50 \% 288.40$ (3.02-27518.50), $-40 \% 154.85$ (1.25-19163.07), $-30 \% 109.03(0.82-14519.39)$, - $20 \% 9.70$ (0.74-127.30). C Percentage change in eGFR over 3 years; $-30 \% 1.27 \times 10^{76}$ $(0-),-20 \% 8.87 \times 10^{25}$ $\left(0-1.90 \times 10^{138}\right) . a H R$ adjusted hazard ratio, $L n(a H R)$ natural logarithm (adjusted hazard ratio), UACR urinary albuminto-creatinine ratio
A

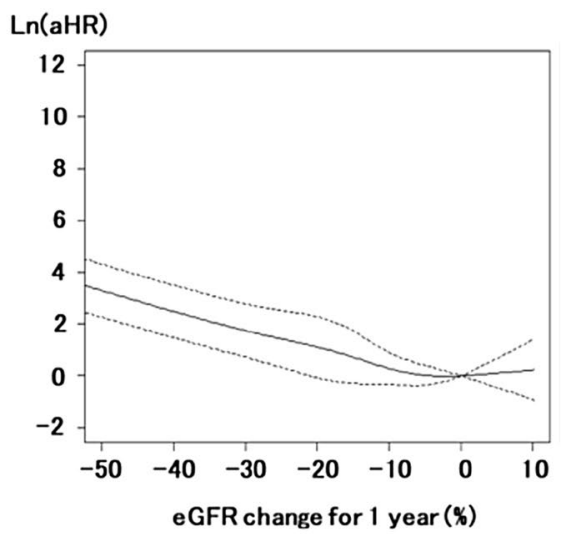

C

$\operatorname{Ln}(\mathrm{aHR})$

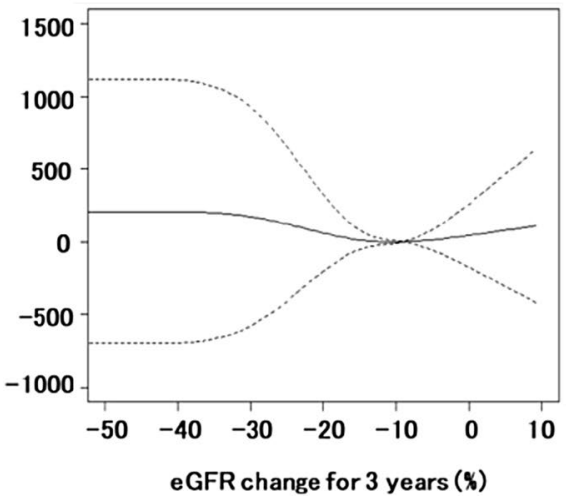

B

$\operatorname{Ln}(\mathrm{aHR})$

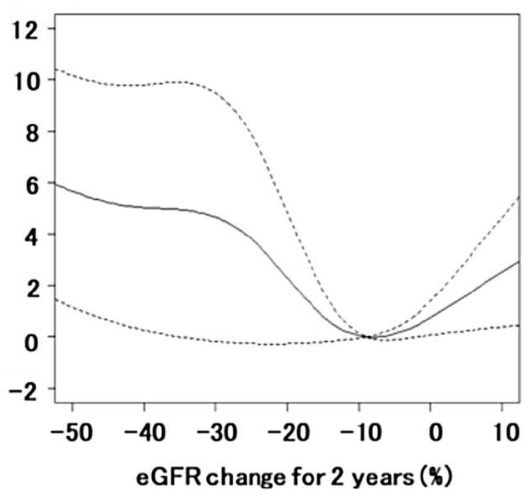

selected as the candidate surrogate endpoints for both the high- and low-eGFR groups in this analysis.

We analyzed the data of the ORIENT study which targeted progressive DKD. In one study, meta-analysis was conducted on the relation between true endpoints and surrogate endpoints from 37 randomized trials, and only the relationships between the risk of ESRD and percentage changes in eGFR over 1 year were investigated. Compared to percentage changes in eGFR of $0,30 \%$ was HR $9.6(95 \%$ CI 7.3-12.6) and 40\% was HR 20.3 (95\% CI 14.1-29.3). Correlation was found between percentage changes in eGFR and risk of ESRD [10]. In this study, the relationships between percentage changes in eGFR and risk of ESRD were investigated. For both percentage changes in eGFR over 1 and 2 years, the greater the decrease in the percentage changes in eGFR, the greater was the tendency toward high risk of ESRD. However, this relationship was not seen for percentage changes in eGFR over 3 years. One reason why correlation between percentage changes in eGFR over 3 years and risk of ESRD was not easily seen in this study may be because percentage changes in eGFR over 3 years were observed only in a few cases, and as a result, the relationship between percentage changes in eGFR over 3 years and risk of ESRD could not be observed accurately. In addition, given that this outcome differed from the data of OGHMA, it suggests that the appropriate baseline period for percentage changes in eGFR differs according to the subject.

Next, the treatment effects were studied. It was found that the aHR of ESRD was less than 1 for percentage changes in eGFR of $20-30 \%$ over 1 year and percentage changes in eGFR of $20-50 \%$ over 2 years. Of this, percentage changes in eGFR over 2 years of $40 \%$ showed the closest aHR to the true endpoint. In a study conducting the meta-analysis of 37 randomized trials, the effects of various treatment methods such as renin-angiotensin-aldosterone system (RAS) inhibitor and calcium channel blocker on true endpoints and surrogate endpoints were compared [36]. According to this study, the evaluation of the HR of RAS inhibitors for true endpoints and that for surrogate endpoints indicated it to be 0.98 (95\% CI $0.89-1.07)$ in the case of percentage changes in eGFR of $40 \%$ over 2 years and 1.08 (95\% CI $0.95-1.20)$ in the case of $30 \%$, demonstrating that $40 \%$ is closer to the true endpoint result than $30 \%$. For this reason, the percentage change in eGFR of $40 \%$ over 2 years was selected as the candidate surrogate endpoint in this analysis. 
aHR
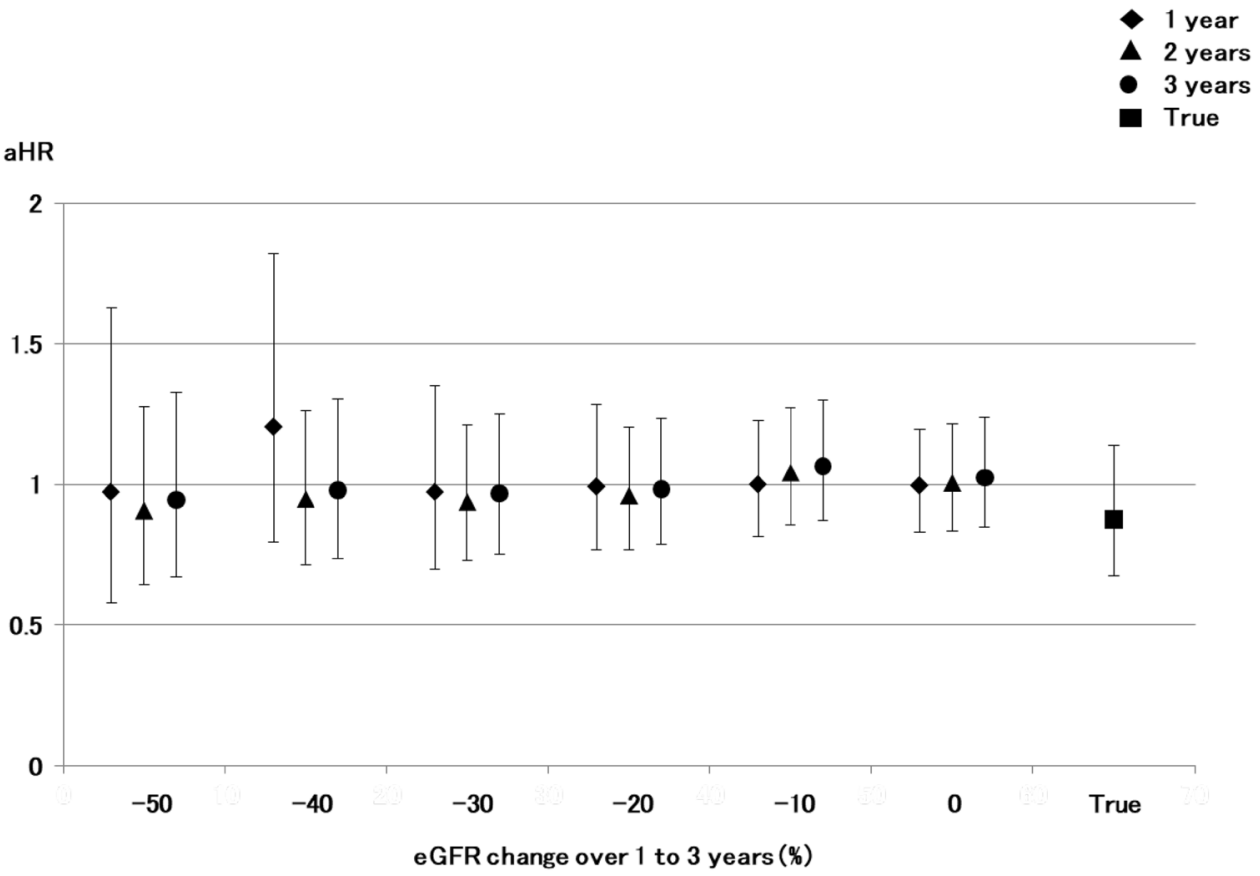

Fig. 15 Effects of olmesartan on endpoints. aHR adjusted for baseline characteristics (eGFR, UACR, and country) and 95\% CI are shown. A Percentage changes in eGFR over 1 year; $-50 \%$ aHR 0.97 (95\% CI 0.58-1.63), - 40\% 1.2 (0.79-1.82), - 30\% 0.97 (0.7-1.35), - $20 \%$ 0.99 (0.77-1.28), - $10 \% 1$ (0.82-1.23), $0 \% 1(0.83-1.2)$. B Percentage changes in eGFR over 2 years; $-50 \% 0.91(0.65-1.28),-40 \%$ 0.95 (0.72-1.26), - 30\% $0.94(0.73-1.21),-20 \% 0.96(0.77-1.2)$,

Taking into account the results of the two analyses in this study and results of studies conducted to date, all subjects showed correlation between percentage changes in eGFR and risk of ESRD, and the greater the decrease in the percentage changes in eGFR, the greater was the tendency towards high risk of ESRD. It has been reported that shortterm changes in eGFR during the administration of RAS inhibitors are not associated with long-term renal failure [37]. The baseline period of 1 year cannot be said to be appropriate for observing renal protective effects and the onset of side effects [10]. The candidate baseline period was 2 years in the studies by Coresh et al., Chang et al., and in the CKD-JAC study [8, 18, 20]. In our studies, it was 2 or 3 years in the analysis of the data of OGHMA, and 2 years in the analysis conducted in the ORIENT study. The joint research group of the US National Kidney Foundation and FDA recommends 2 or 3 years [12]. Thus, the candidate baseline period of percentage changes in eGFR for surrogate endpoints differs according to the subject, and may be 2 or 3 years.

On the other hand, the candidate cutoff level was $30 \%$ in the study by Coresh et al., $30 \%$ in that by Chang et al., and 30 or $40 \%$ in the CKD-JAC study [8, 18, 20]. In our studies, it was $30 \%$ or $40 \%$ in the analysis of the data of health
- 10\% 1.04 (0.86-1.28), $0 \% 1.01$ (0.83-1.22). C Percentage changes in eGFR over 3 years; - 50\% $0.95(0.67-1.33),-40 \% 0.98(0.74$ $1.3),-30 \% 0.97(0.75-1.25),-20 \% 0.99(0.79-1.23),-10 \% 1.06$ (0.87-1.3), $0 \% 1.03$ (0.85-1.24). True endpoint; 0.88 (0.68-1.14). $a H R$ adjusted hazard ratio, True true endpoint throughout study period, UACR urinary albumin-to-creatinine ratio

examinations conducted in Okinawa, Japan, and $40 \%$ in the analysis of the ORIENT study. The joint research group of the US National Kidney Foundation and FDA recommends 30 and $40 \%$ [37]. The candidate cutoff level of surrogate endpoints differs according to the subject, and may be 30 or $40 \%$. The doubling of the serum creatinine level used as the conventional ESRD surrogate endpoint is equivalent to $53 \%$ decrease in eGFR, when converted to percentage change in eGFR based on the eGFR estimation equation of the Japanese Society of Nephrology. Moreover, the percentage changes in eGFR of 30 and $40 \%$ were 1.4 times and 1.6 times the respective serum creatinine levels. In the study by Coresh et al., comparison of the $57 \%$ percentage change in eGFR (which is equivalent to the doubling of the serum creatinine levels used in the US) and 30\% indicated that there were only a few subjects with decrease in percentage change in eGFR of more than 57\% [8]. Here, there are two percentage changes in eGFR that are equivalent to the doubling of the serum creatinine levels, 53 and 57\%, due to the mathematical differences between the eGFR estimation equation of the Japanese Society of Nephrology for Japanese and CKD Epidemiology Collaboration (CKD-EPI) equation used in Europe and the US. In this study, subjects with decrease in percentage changes in eGFR of more than 


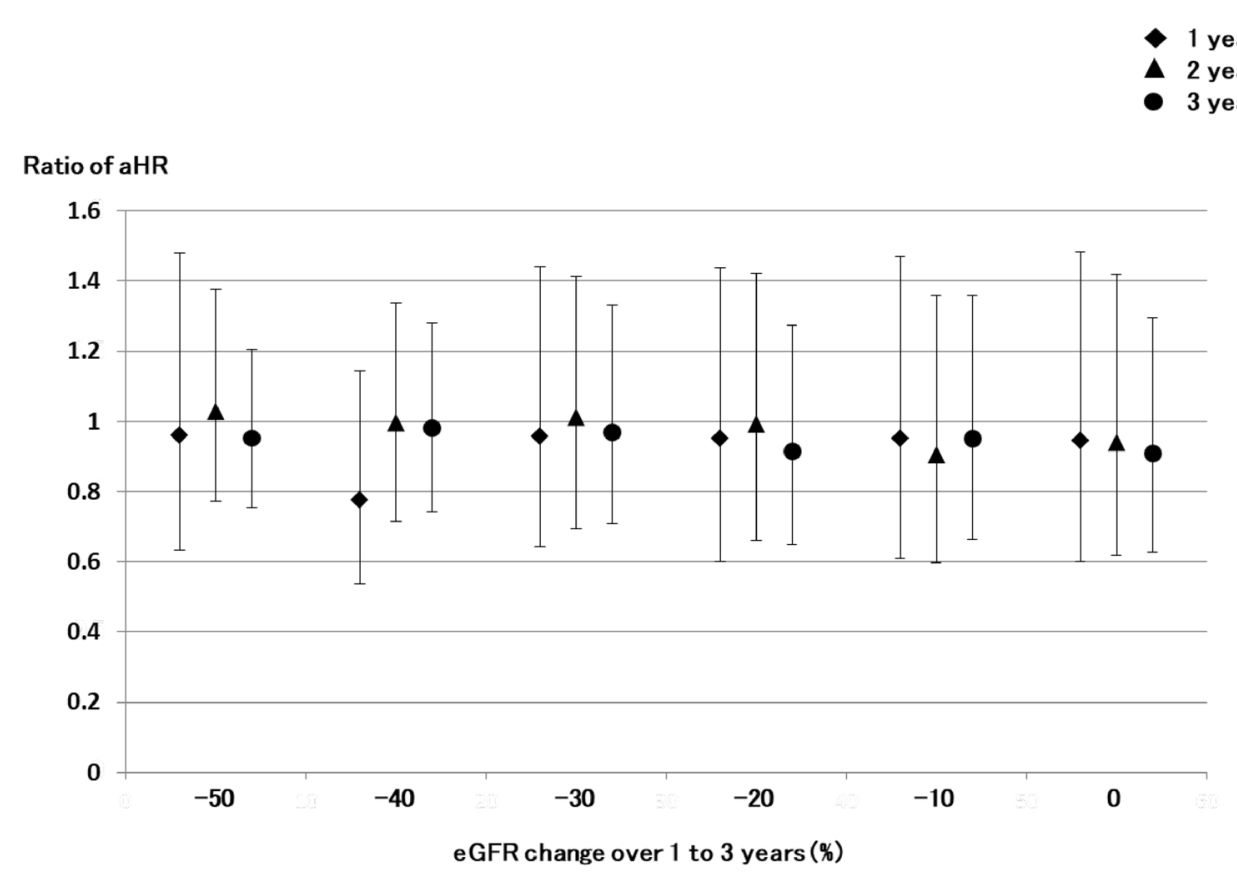

Fig. 16 Comparison of effects of olmesartan on endpoints. The median ratio and $95 \% \mathrm{CI}$ of the comparison of the effects (aHR) of olmesartan on true endpoints and surrogate endpoints are shown. Adjusted for baseline characteristics (eGFR, UACR, and country). Percentage changes in eGFR over 1 year; $-50 \%$ aHR comparison 0.96 (95\% CI 0.63-1.48), - 40\% 0.78 (0.54-1.15), - 30\% 0.96 (0.64-1.44), - $20 \% 0.95$ (0.6-1.44), - 10\% 0.95 (0.61-1.47), $0 \%$ 0.95 (0.6-1.48). Percentage changes in eGFR over 2 years; $-50 \%$

$50 \%$ showed a tendency for greater risks of ESRD compared to those with 30 or $40 \%$, but subjects with more than $50 \%$ were rare. The faster the decrease in the percentage changes in eGFR, the greater was the risk of ESRD, but the fewer was the number of such subjects. In a sample size estimation simulation study, comparison of the sample sizes with percentage changes in eGFR of 30 or $40 \%$ as the surrogate endpoint and with $57 \%$ as the surrogate endpoint showed the sample size of 30 or $40 \%$ to be smaller than the samples size of $57 \%$ by more than $20 \%$ [11].

Candidate surrogate endpoints obtained in this study showed varied baseline periods and cutoff levels. Given that the baseline period and cutoff level of the appropriate percentage changes in eGFR may differ according to the subject, there is a need to evaluate various subjects and treatment methods when designing a study, and the need to set appropriate surrogate endpoints. Since this study was mostly based on the data of health examinations of healthy persons and on the retrospective analysis of relatively small-scale $\mathrm{RCT}$, it is not clear if the results obtained in this study can broadly be applied to CKD patients in Japan. In addition, given that the percentage changes in eGFR are calculated from serum creatinine levels, they are affected by muscle mass, nutritional state, and creatinine excretion amount,
$1.03(0.77-1.38),-40 \% 1.0(0.71-1.34),-30 \% 1.01(0.69-1.41)$, - $20 \% 0.99(0.66-1.42),-10 \% 0.91(0.6-1.36), 0 \% 0.94(0.62-$ 1.42). Percentage changes in eGFR over 3 years; $-50 \% 0.95(0.75$ $1.2),-40 \% 0.98(0.74-1.28),-30 \% 0.97(0.71-1.33),-20 \% 0.91$ $(0.65-1.28),-10 \% \quad 0.95 \quad(0.66-1.36), 0 \% 0.91 \quad(0.63-1.3)$. $\quad$ aHR adjusted hazard ratio, True true endpoint throughout study period, $U A C R$ urinary albumin-to-creatinine ratio

which may cause eGFR estimation errors [35]. Moreover, since errors can occur easily in elderly persons and cases with complications such as malnutrition and chronic inflammation, it is advisable to also evaluate renal functions using methods based on biomarkers other than serum creatinine. If subjects include patients with complications such as cardiovascular diseases or elderly persons, competing endpoints other than ESRD such as death and cardiovascular diseases can occur [10]. In such cases, there is a need to evaluate composite endpoints taking into account these endpoints.

\section{Conclusion}

In this study, the usefulness of the percentage change in eGFR as the surrogate endpoint of ESRD was demonstrated. The outcome of this study and studies conducted to date suggests that decreases in percentage changes in eGFR of 30 or $40 \%$ over 2 or 3 years may be candidate surrogate endpoints for ESRD. Moreover, given that the baseline period and cutoff level of the appropriate percentage change in eGFR differ according to the subject, there is a need to set appropriate surrogate endpoints when designing studies. 


\section{Systematic review}

\section{CQ1: What is the appropriate level of decline in GFR as a surrogate endpoint of end-stage renal disease?}

\section{Background}

In clinical trials on CKD drugs to date, progression to ESRD or initiation of renal replacement therapy (dialysis or transplantation) has been widely used as a hard endpoint. However, reaching these hard endpoints can take considerable time depending on the baseline renal function because there are only a few events. For this reason, large-scale clinical trials with long observational period are required. Furthermore, as renal replacement therapy initiation criteria are not standardized, the hard endpoints themselves have been called inconsistent. The registration and long-term follow-up of a large number of patients to achieve these hard endpoints require enormous time and costs, making it difficult for new CKD drugs to be developed. To promote the development of new CKD drugs, surrogate endpoints allowing fast assessments and highly predictive of progression to ESRD are essential. One surrogate endpoint which has already been established is the time required for the doubling of serum creatinine level. The doubling of the serum creatinine level has already been reported in a clinical study conducted in the 1970s and has been applied widely since then. However, according to the analysis by CKD-EPI, the doubling of the serum creatinine level is equivalent to 57\% eGFR decline, and is considered as a relatively late event. In this study, we thus searched literature to determine if even more moderate decrease in eGFR can be used as a surrogate endpoint.

\section{Literature search}

Literature was searched in Pubmed between January 1970 and December 2016.

Search terms used were (decline) (glomerular filtration rate) (end-stage renal disease) (end-stage kidney disease). Nine hundred and thirty-three papers were found using the search expressions "(glomerular filtration rate) AND [(endstage renal disease) OR (end-stage kidney disease)] AND (decline)". Titles and abstracts were first reviewed to select applicable articles, these were then reviewed a second time (the entire paper was reviewed), and a total of nine papers met this CQ.

Details of search expressions

- ("glomerular filtration rate"[MeSH Terms] OR ("glomerular"[All Fields] AND "filtration"[All Fields] AND "rate"[All Fields]) OR "glomerular filtration
rate"[All Fields]) AND ((“kidney failure, chronic"[MeSH Terms] OR ("kidney"[All Fields] AND "failure"[All Fields] AND "chronic"[All Fields]) OR "chronic kidney failure"[All Fields] OR ("end"[All Fields] AND "stage"[All Fields] AND "renal"[All Fields] AND "disease"[All Fields]) OR "end-stage renal disease"[All Fields]) OR ("kidney failure, chronic"[MeSH Terms] OR ("kidney"[All Fields] AND "failure"[All Fields] AND "chronic" [All Fields]) OR "chronic kidney failure"[All Fields] OR ("end"[All Fields] AND "stage"[All Fields] AND "kidney"[All Fields] AND "disease"[All Fields]) OR "end-stage kidney disease" [All Fields])) AND decline[All Fields] AND ("1970/01/01”[PDAT]: “2016/12/31”[PDAT]).

\section{Discussion}

Observational study on Stages 3 and 4 of CKD Meta-analysis using the data of 35 cohorts and 1,700,000 cases of the CKD Prognosis Consortium (CKD-PC), a large-scale international epidemiological study composed of several Japanese cohorts, indicated the frequency of decrease in eGFR of 30 and $40 \%$ and HR of ESRD risk for 2- and 3-year baseline periods. In patients with a baseline eGFR of less than $60 \mathrm{ml} / \mathrm{min} / 1.73 \mathrm{~m}^{2}$ (mean eGFR $48 \mathrm{~mL} / \mathrm{min} / 1.73 \mathrm{~m}^{2}$ ), the decrease in eGFR of more than $30 \%$ in the 2-year baseline period was $6.9 \%$ (95\% CI 6.4-7.4), that of more than $40 \%$ was $3.2 \%(2.8-3.7)$, and that of more than 57 was $0.79 \%(0.52-1.06)$. Compared to the decrease in eGFR of $0 \%$, the HR of ESRD risk over 2 years was 5.4 (95\% CI 4.5-6.4) for 30\% decrease in eGFR, $10.2(8.2-12.7)$ for $40 \%$ decrease, and 32.1 (22.3-46.3) for 57\% decrease. On the other hand, the decrease in eGFR of more than $30 \%$ in the 3 -year baseline period was 9.5\% (95\% CI 8.9-10.2), that of more than 40 was $4.8 \%$ (4.3-5.4), and that of more than 57 was $1.3 \%$ (0.9-1.7). Moreover, the HR of ESRD risk over 3 years was 5.0 (95\% CI 3.9-6.4) for 30\% decrease in eGFR, 10.4 (8.0-13.4) for 40\% decrease, and 36.8 (27.3-49.7) for $57 \%$ decrease. In patients with a baseline eGFR of $35 \mathrm{~mL} /$ $\min / 1.73 \mathrm{~m}^{2}$, the risk of progression to ESRD in 10 years (revised using covariate and competing risk of death) was 99\% (95\% CI 95-100\%) for 57\% decrease in eGFR over 2 years, $83 \%$ (71-93\%) for $40 \%$ decrease, $64 \%$ (52-77\%) for $30 \%$ decrease, and $18 \%$ (15-22\%) for $0 \%$ decrease [8].

In a meta-analysis of 37 randomized controlled trials on CKD patients (9488 subjects, mean age $52 \pm 13$ years, baseline eGFR $49.2 \pm 24.9 \mathrm{ml} / \mathrm{min} / 1.73 \mathrm{~m}^{2}$ ), the predictive ability of already established endpoints (ESRD, eGFR $<15 \mathrm{~mL} /$ $\min / 1.73 \mathrm{~m}^{2}$, doubling of serum creatinine level) was evaluated for patients where 30 or $40 \%$ decrease in eGFR was observed over 12 months. In the 12-month baseline period, eGFR decreased by more than 30 in $16.1 \%$ patients, and by more than 40 in $7.8 \%$ patients. After the 12-month baseline 
period, further follow-up (median 2 years) revealed that 2661 patients reached the endpoint. Compared to the decrease in eGFR of $0 \%$, the HR for reaching the endpoint was $9.6(95 \%$ CI 7.3-12.6) for 30\% or more decrease in eGFR, $20.3(95 \%$ CI 7.3-12.6) for $40 \%$ or more. This relationship was seen regardless of the baseline eGFR, proteinuria, underlying disease, and treatment [10].

In the observational study conducted on Stages 3 and 4 CKD patients in Japan, the contribution of the following terms to the progression to ESRD (initiation of hemodialysis or peritoneal dialysis) as an endpoint was reviewed, namely baseline eGFR, proteinuria, underlying disease, age, mean albumin, hemoglobin, phosphorus, and proteinuria during the 2-year baseline period, and percentage decrease in eGFR during the 2-year baseline period. During the followup period $(4.5 \pm 1.3$ years $), 83$ out of the 701 subjects progressed to ESRD. As for contribution to the progression to ESRD, baseline eGFR and proteinuria were the most influential factors in the laboratory findings for baseline and mean values during the observational period. With every $10 \%$ increase in the percentage decrease in eGFR during the 2-year baseline, the HR of the risk of ESRD increased exponentially. Compared to the 0 to $10 \%$ decrease in eGFR, the HR of the progression to ESRD was 38.9 (95\% CI 5.1-298.1) for the 30 to $40 \%$ decrease in eGFR. In the laboratory findings for baseline, the $30 \%$ decrease in eGFR showed the highest HR for progression to ESRD (HR 31.6), instead of the mean albumin, hemoglobin, phosphorus, and proteinuria. With the Cox proportional hazards model, model fitness improved when baseline eGFR, proteinuria, albumin, hemoglobin were added [18].

These findings suggest that in CKD Stages 3 and 4 patients, it may be possible to use 30 or $40 \%$ decrease in eGFR over 2 or 3 years as a surrogate endpoint by the prediction of the progression to ESRD thereafter.

\section{Observational studies mainly targeting non-CKD and stages}

1 and 2 of CKD In the atherosclerosis risk in communities (ARIC) study, which is a large-scale prospective observational study on the general population, 9703 subjects who could be followed up for a long period of time were investigated with regard to the relationship between progression to ESRD (initiation of dialysis and renal transplantation) and changes in eGFRcr (creatinine) calculated using the CKDEPI equation, eGFRcys (cystatin C) and $\beta 2$-microglobulin reciprocal (1/B2M) over 6 years. The results showed that the 10 to $30 \%$ decrease and more than $30 \%$ decrease in eGFRcr, eGFRcys, and 1/B2M over 6 years are useful for predicting the risks of progression to ESRD later. HR was 2.6 for 10 to $30 \%$ decrease in eGFRcr, 20.0 for a decrease of more than $30 \%$; 3.0 for 10 to $30 \%$ decrease in eGFRcys, 16.7 for a decrease of more than $30 \%$; 4.0 for 10 to $30 \%$ decrease in $1 / \mathrm{B} 2 \mathrm{M}$, and 22.5 for a decrease of more than $30 \%$. When the mean values of the three terms decreased by more than $30 \%$, risks of ESRD were even higher than eGFRcr alone (HR 32.0) [32].

Investigations on the changes in renal functions in shorter time than 2 years and progression to ESRD have also been reported. Of the CKD Stages 1-4 patients ( $86 \%$ above Stages 1 and 2) above 18 years old registered with the Alberta Kidney Disease Network, 598,397 subjects whose eGFR was measured twice in 6 months- 1 year were investigated, and adjustments were carried out in the HR using the first available eGFR. Compared to the group with no change in eGFR, the group with more than $25 \%$ decrease in eGFR showed increase in the HR of ESRD by 5.1 times (95\% CI 4.6-5.7). However, when HR was adjusted with the last eGFR at baseline period and covariate, the HR of ESRD did not increase in the group with more than $25 \%$ decrease in eGFR. It has been concluded that change in eGFR (more than 25\% decrease in eGFR) should be used for predicting risks of ESRD based on the measurement of serum creatinine level more than twice after at least more than 1 year [31].

Furthermore, a follow-up study was conducted on 161 subjects with type $1 \mathrm{DM}$ with proteinuria and $\mathrm{eGFR} \geqq 60 \mathrm{ml} /$ $\mathrm{min} / 1.73 \mathrm{~m}^{2}$ (CKD Stages 1 and 2) for $5-18$ years to investigate the changes in eGFR and risks of ESRD using serum creatinine levels measured for 5 times continuously, the tendency for decrease in GFR for $5 \mathrm{ml} / \mathrm{min} / 1.73 \mathrm{~m}^{2} /$ year in 5 years after the first baseline is able to predict progression to ESRD after that than glycosylated hemoglobin, blood pressure, and urine albumin/gCr ratio [38].

In non-CKD or CKD Stage 1 or 2 patients, decrease in eGFR over a long period such as 5 or 6 years may be able to predict progression to ESRD. These reports suggest that it is difficult to define the observational period and percentage change in eGFR, and set the surrogate endpoint.

Interventional studies Investigations comparing the effects of CKD intervention between established endpoints (ESRD, eGFR $<15 \mathrm{~mL} / \mathrm{min} / 1.73 \mathrm{~m}^{2}$, doubling of serum creatinine levels) and new surrogate endpoints (decrease in eGFR of $57,40,30,20 \%$ over $1-3$ years)

Investigations were conducted on the 3228 adult patients with type 2 diabetic nephropathy (RENAAL mean Cr $1.9 \mathrm{mg} / \mathrm{dl}$, mean eGFR $41.4 \mathrm{ml} / \mathrm{min} / 1.73 \mathrm{~m}^{2}$, IDNT mean $\mathrm{Cr} 1.7 \mathrm{mg} / \mathrm{dl}$, mean eGFR $50.2 \mathrm{ml} / \mathrm{min} / 1.73 \mathrm{~m}^{2}$ ) who participated in the Reduction in End Points in Noninsulin-Dependent Diabetes Mellitus with the Angiotensin II Antagonist Losartan (RENAAL) study which verified the treatment effects of Angiotensin II Receptor Blocker (ARB) and Irbesartan Diabetic Nephropathy Trial (IDNT) to compare the predictive ability of ESRD composite endpoints, namely doubling of serum creatinine levels at months $12,18,24$, and 36 and 57, 40,30, and $20 \%$ decreases in eGFR, or eGFR $<15 \mathrm{ml} / \mathrm{min} / 1.73 \mathrm{~m}^{2}$, as well 
as evaluate the number of patients reaching the endpoints, inconsistencies in ARB treatment effects, and statistical significance. When $20 \%$ decrease in eGFR was used, there were many patients who reached the endpoints, and inconsistencies in treatment effects decreased; however, statistical significance decreased [39]. ARB treatment effects were compared between use of eGFR before the start of ARB treatment and use of eGFR 3 months after start as the baseline kidney function. ARB treatment effects were evaluated as low (decrease in treatment effects) when eGFR before start of treatment was used compared to that 3 months after start. When the decrease in eGFR was less than the doubling of the serum creatinine level, the statistical power of clinical studies did not improve due to the reduction of treatment effects, even though inconsistencies in treatment effects decreased. The reduction of treatment effects is thought to be partially due to the acute effects of ARB on eGFR. The Discussion section of this paper states that when more moderate decrease in eGFR (namely 20, $30,40 \%$ ) are used as surrogate endpoints in clinical studies, there is a need to take into account temporary decrease in GFR due to the reduction of glomerular perfusion as a result of ARB treatment intervention, and set the run-in period after treatment instead of setting the start of the evaluation of GFR decrease immediately after the start of treatment.

Inker et al. [40] conducted a meta-analysis of 37 randomized controlled trials on CKD patients (9488 subjects) where they analyzed five treatment interventions (RAS inhibition versus control, RAS inhibition versus calcium antagonist, aggressive blood pressure management, protein-restricted diet, immunosuppressive therapy) and CKD progression, and evaluated surrogate endpoints 20, 30, 40, and $57 \%$ decreases in eGFR during the baseline periods of 12,18 , and 24 months by their predictive ability for already established endpoints (ESRD, eGFR $<15 \mathrm{~mL} / \mathrm{min} / 1.73 \mathrm{~m}^{2}$, doubling of serum creatinine levels). Except for proteinrestricted diet intervention for which strong treatment effects were observed, reduction of treatment effects was seen for surrogate endpoints. The HR (95\% CI) of the respective surrogate endpoints and established endpoints of the five treatment interventions was between $0.91(0.64-1.43)$ and 1.12 $(0.89-1.40)$ in respect to the $40 \%$ decrease in eGFR, and between $0.88(0.63-1.39)$ and $1.15(0.88-1.54)$ in respect to the $30 \%$ decrease in eGFR. Thus, it was concluded that the 30 or $40 \%$ decrease in eGFR can be used as surrogate endpoints, but the use of $40 \%$ decrease is supported over $30 \%$ decrease [40]. In the "Discussion" section of this paper, reasons for the changes in HR observed when decrease in eGFR is used as an endpoint include decrease in eGFR (negative acute effects) by RAS inhibition, aggressive blood pressure management, protein-restricted diet, allometric effects of treatment intervention on decrease in eGFR, and influence of protein-restricted diet intervention on the production of creatinine.

Furthermore, simulation studies reviewing the risks of type 1 error (false positive) and sample sizes when decrease in eGFR is used as a surrogate endpoint have been reported. Although the use of endpoints based on the 30 or $40 \%$ decrease in eGFR is an appropriate strategy for reducing sample size in specific circumstances, the risks of type 1 error increase when there are acute effects, especially when eGFR decreases by $30 \%$. It was concluded that use of these endpoints must be decided after evaluating the anticipated effects (decrease in sample size, improvement of statistically detection ability) under specific clinical study conditions [11].

From the results of these interventional studies and simulation studies, when the effects of intervention for CKD are evaluated using surrogate endpoints (30 or $40 \%$ decrease in eGFR over 2 or 3 years), in intervention which may lead to temporary decrease in eGFR, it was concluded that there is a need to carefully set surrogate endpoints by setting a certain run-in period after treatment, and to evaluate the use of 30 or $40 \%$ percentage decrease in eGFR, instead of setting the start of evaluation immediately after the start of treatment.

\section{Conclusion}

In CKD Stages 3 and 4 patients, the 30 or $40 \%$ decrease in eGFR over 2 or 3 years may be useful surrogate endpoints of progression to ESRD. Given that the appropriate observational period and percentage decrease in eGFR may differ according to the research subject, there is a need to set surrogate endpoints according to the research subject. When using surrogate endpoints for the evaluation of intervention which may cause temporary decrease in eGFR, there is a need to carefully set the start time of the observational period and select the percentage decrease in eGFR.

\section{CQ2: Is decrease in albuminuria or proteinuria an appropriate surrogate endpoint for replacing prevention of progression to ESRD?}

\section{Background}

Albuminuria/proteinuria is an independent risk factor of ESRD [41]. In addition, in interventional studies, changes in albuminuria/proteinuria due to treatment have been reported to be also associated with outcomes such as ESRD, doubling of serum creatinine levels, and death [42-46]. Consequently, there are reports recommending use of the decrease in albuminuria/proteinuria as the surrogate endpoint in interventional studies $[47,48]$. On the other hand, there are reports concluding that it is an inappropriate surrogate endpoint [49], and reports which state that although 
there exists sufficient evidence for concluding that decrease in proteinuria is a useful surrogate endpoint only for diabetic nephropathy and only when RA inhibitor is used in CKDs other than diabetic nephropathy [50], indicating a division of opinions. Thus, in this CQ, we investigated if the decrease in albuminuria/proteinuria is an appropriate surrogate endpoint for replacing the prevention of progression to ESRD.

\section{Literature search}

Literature was searched in Pubmed between January 1970 and September 2016. Search terms used were [(proteinuria) OR albuminuria)] AND [(kidney failure) OR renal failure]. Eight hundred and sixty-three papers were found using the following search expressions, and their titles and abstracts were first reviewed. Applicable articles were selected and reviewed a second time (the entire paper was reviewed). By adding some hand search, a total of 10 papers meeting this CQ were selected.

(("renal insufficiency"[MeSH Terms] OR ("renal"[All Fields] AND "insufficiency"[All Fields]) OR "renal insufficiency"[All Fields] OR ("kidney"[All Fields] AND "failure"[All Fields]) OR "kidney failure"[All Fields]) OR ("renal insufficiency"[MeSH Terms] OR ("renal"[All Fields] AND "insufficiency"[All Fields]) OR "renal insufficiency"[All Fields] OR ("renal"[All Fields] AND "failure"[All Fields]) OR "renal failure"[All Fields])) AND (("proteinuria"[MeSH Terms] OR "proteinuria”[All Fields]) OR ("albuminuria"[MeSH Terms] OR "albuminuria"[All Fields])) AND ((Meta-Analysis[ptyp] OR Randomized Controlled Trial[ptyp] OR systematic[sb]) AND (“0001/01/01”[PDAT]: “2016/09/31”[PDAT])).

\section{Discussion}

As evidence of this CQ, albuminuria/proteinuria at baseline and at the end of the study was measured, and six RCT articles [42-44, 51-53] and four meta-analysis articles [45, 46, 54, 55] using ESRD as the endpoint were adopted.

In the RENAAL study verifying the renal protection effects of Losartan on patients with diabetic nephropathy and overt proteinuria, the relationship between changes in albuminuria 6 months after the start of treatment based on baseline and ESRD risk was evaluated [42]. Albuminuria decreased $28 \%$ in the Losartan group based on baseline, and increased $4 \%$ in the placebo group. Given that ESRD risk decreased $45 \%$ with every decrease in albuminuria of $50 \%$, the relative risk of albuminuria reduction effects and onset of ESRD showed a more or less linear relationship. Likewise, in a post hoc analysis of IDNT study administering Irbesartan, Amlodipine, or placebo to patients with diabetic nephropathy and overt proteinuria, the relationship between changes in proteinuria 12 months later and composite endpoint (doubling of serum creatinine levels, or more than $6.0 \mathrm{mg} / \mathrm{dL}$, ESRD) was evaluated [43]. The percentage decrease in the mean proteinuria 12 months after the start of treatment was $41 \%$ in the Irbesartan group, $11 \%$ in the amlodipine group, and $16 \%$ in the placebo group. The risks of composite renal endpoints decreased to below half each time the proteinuria 12 months after the start of treatment decreased by $50 \%$ (HR $0.44 ; 95 \%$ CI $0.40-0.49$ ) [43]. In a joint review of the ongoing Telmisartan alone and in combination with Ramipril global endpoint trial (ONTARGET) and Telmisartan Randomised AssessmeNt Study in ACE iNtolerant subjects with cardiovascular Disease (TRANSCEND) conducted on patients with arteriosclerotic cardiovascular disease or DM patients with organ damage, compared to the groups showing changes in albuminuria 2 years later of an increase of less than double or decrease of up to $50 \%$, the HR of composite endpoints (doubling of serum creatinine levels or initiation of dialysis) was 1.4 (95\% CI 1.11-1.78) in the group showing increase of more than $100 \%$, and 0.73 (95\% CI $0.57-0.95$ ) in the group showing decrease of more than $50 \%$. To determine changes in albuminuria, renal endpoints were predicted regardless of the baseline albuminuria [44]. These randomized controlled trials and post hoc analysis results confirmed a relationship between the decrease in albuminuria/proteinuria as a result of treatment using RA inhibitors for diabetic nephropathy and prevention of progression to ESRD. On the other hand, there are four papers reporting systematic review/ meta-analysis reviewing the usefulness of decrease in albuminuria/proteinuria as a surrogate endpoint $[45,46,54$, 55]. In a meta-analysis of 32 randomized controlled trials (9008 subjects) evaluating five treatment interventions (RA inhibitor, $\mathrm{Ca}$ antagonist, intensive depressor therapy, low protein diet, and immunosuppressive drug) with renal disease progression as an outcome, the relationships between changes in albuminuria 2.5-13 months later and composite endpoints (doubling of serum creatinine level, ESRD, death) were investigated [45]. In patients with decreased proteinuria, the risks of reaching the endpoint were low, the total HR per decrease in albuminuria of 50\% was 0.74 (95\% CI $0.67-0.82)$. In a meta-analysis of 21 studies $(78,342$ subjects) investigating the effects of RA inhibitor, intensive depressor therapy, dyslipidemia drug, low-protein diet, etc. on ESRD, the median observational period of changes in albuminuria was 6 months, and the treatment effects for albuminuria showed a significant correlation with the treatment effects for ESRD. Each time albuminuria decreased by $30 \%$, ESRD risks decreased by $23.7 \%$ (95\% CI $11.4-34.2 \%$ ) [46]. On the other hand, in a meta-analysis of 11 randomized controlled trials (830 subjects) investigating the effects of RA inhibitor, fish oil, immunosuppressor, and steroid treatment for intensive depressor therapy, dyslipidemia drug, low protein diet, etc. on IgA nephropathy, the decrease in 
proteinuria 9 months after the start of treatment was related to the decrease in the risk of composite renal endpoint (doubling of serum creatinine level, ESRD, death), and the HR with every decrease in proteinuria of 50\% was 0.40 (95\% CI $0.32-0.48)$. Here, the changes in proteinuria of RA inhibitors and corticosteroids matched the course of treatment effects for composite endpoints. It has also been reported that when using RA inhibitors and corticosteroids for IgA nephropathy, the early decrease in proteinuria is a useful surrogate endpoint [54]. Furthermore, in a meta-analysis of 27 randomized controlled trials $(97,458$ subjects) with ESRD as the outcome, the validity of the two surrogate endpoints, changes in proteinuria and doubling of serum creatinine levels, was verified using the index, treatment effect ratio (TER). TER is the ratio of the treatment effects for ESRD and changes in surrogate endpoints. It indicates that when TER is close to 1, the treatment effects for ESRD and changes in the surrogate endpoint will match. As a result, the TER of the doubling of the serum creatinine level obtained from 20 RCTs (95,457 subjects, 3850 ESRD events) was 0.98 (95\% CI $0.85-1.14$ ), more or less matching the treatment effects for ESRD. This indicates that the doubling of the serum creatinine level is an excellent surrogate endpoint. The TER of proteinuria was verified from seven randomized controlled trials (17,740 subjects, 173 ESRD events) including four randomized controlled trials conducted on subjects with diabetic nephropathy accompanied by overt proteinuria. Specifically, there were five treatment interventions using RA inhibitors, one using dyslipidemia drug and one using EDTA chelating agent. As a result, the TER of proteinuria was 0.82 (95\% CI 0.59-1.16), and changes in proteinuria were related to the treatment effects of ESRD. Since little data were obtained, it was concluded that there is a limit to evidence as a surrogate endpoint [55]. The results of these meta-analyses demonstrate that the decrease in albuminuria/proteinuria can be used as a surrogate endpoint for the progression of renal disease, not only for RA inhibitor for diabetic nephropathy accompanied by overt proteinuria but also treatment using RA inhibitor and corticosteroid for IgA nephropathy. However, it indicated that compared to the doubling of serum creatinine level, evidence is limited.

When decrease in albuminuria/proteinuria is used as a surrogate endpoint, is it possible to clearly set the percentage increase and observational period? In a post hoc analysis of the Olmesartan Reducing Incidence of Endstage Renal Disease in Diabetic Nephropathy Trial (ORIENT) administering olmesartan or placebo to 566 type 2 DM patients with overt albuminuria, the mean percentage change in proteinuria 24 weeks after the start of treatment was $-19.3 \%$ in the olmesartan group and $+5.1 \%$ in the placebo group. The HR of composite renal endpoints (ESRD, doubling of serum creatinine level, death) comparing the two groups combined and group with increase in proteinuria was 0.54 (95\% CI $0.39-0.74)$ in the group with $0-29 \%$ decrease, and 0.43 (95\% CI $0.31-0.61)$ in the group with decrease of more than $30 \%$. The $30 \%$ decrease in proteinuria 24 weeks after the start of treatment and achievement of remaining proteinuria $<1.0 \mathrm{~g} / \mathrm{gCr}$ were related to renal prognosis [51]. In the post hoc analysis of the Aliskiren Trial in Type 2 Diabetes Using Cardiorenal Endpoints (ALTITUDE) administering Aliskiren or placebo to 8561 type $2 \mathrm{DM}$ patients with chronic renal disease or cardiovascular disease, the median decrease in albuminuria 6 months later was $12 \%$ in the Aliskiren group and $0.0 \%$ in the placebo group [52]. When both groups are combined, the decrease in albuminuria by more than $30 \% 6$ months later resulted in $62 \%$ decrease in composite endpoints (ESRD, serum Cre $>6.0 \mathrm{mg} / \mathrm{dL}$ continuing for 1 month or longer, doubling, death). In both the ORIENT and ALTITUDE trials as well as this study, it was not possible to decrease composite endpoints even through albuminuria was decreased. In all post hoc analyses of treatment groups and placebo groups, the decrease in albuminuria by more than $30 \%$ led to the significant decrease in the composite endpoints. The results of these investigations indicate that significant difference in the percentage decrease in albuminuria/proteinuria between the treated group and control group is insufficient, and a difference of more than $30 \%$ is required. The observational period of albuminuria/proteinuria varies according to the report from 3 months to 2 years. Using the data of 1070 subjects of the RENAAL and IDNT trials, a study was conducted on the relationships between the variability of albuminuria and renal outcome prediction of every patient responding to RA inhibitors [53]. Compared to the 0.68 albuminuria area under the receiver operating characteristic curve of 3 months after the start of treatment (95\% CI 0.64-0.72), that 12 months after was 0.78 (95\% CI 0.75-0.82), indicating significantly high renal outcome predictive ability $(P<0.001)$ (Note 1$)$. In addition, it was suggested that 12-24 months is an appropriate observational period for changes in albuminuria/proteinuria; however, there is a need to accumulate evidence in the future (Note 2). Because albuminuria/proteinuria levels often differ between spot urine in outpatient visits and first morning urine even though they are taken on the same day, ideally the measurement time and method, etc. should be standardized for each study.

Summarizing the above results, RA inhibitor treatment for diabetic nephropathy with overt proteinuria and RA inhibitor or corticosteroid treatment for IgA nephropathy indicated that decrease in albuminuria/proteinuria may be a useful surrogate endpoint for replacing prevention of progression to ESRD. In particular, in diabetic nephropathy with overt proteinuria, there are many papers showing that decrease in albuminuria by more than $50 \%$ from the 
baseline is significant. There are also papers suggesting the potentials of using decrease in albuminuria/proteinuria of more than $30 \%$ from the baseline 12 months after the start of treatment can be used as the surrogate endpoint. However, evidence is insufficient when eGFR is used as an indicator, and also in early CKD. Consequently, at this point, it is not appropriate to generally use decrease in albuminuria/proteinuria as a surrogate endpoint for replacing suppression of progressions to ESRD. There is a need to continue accumulating and further investigating evidence.

\section{Conclusion}

In nephropathy accompanied by proteinuria, the decrease in albuminuria/proteinuria is feasible as a surrogate endpoint for replacing prevention of progression to ESRD. However, there is a lack of evidence when compared to use of eGFR as a parameter.

Note 1 This research group investigated the validity of the changes in proteinuria as a surrogate endpoint for ESRD using the data of OGHMA [56]. Although the findings suggest that changes in proteinuria over 2 years according to the test paper method may serve as a significant predictive factor of ESRD risks even after adjusting with confounders, it was not included in this systematic review due to the timing of the acceptance of this paper.

Note 2 This CQ is planned for review by the workshop on the changes in albuminuria and GFR changes as endpoints of early CKD clinical studies jointly hosted by the National Kidney Foundation, European Medicines Agency, and US Food and Drug Administration on March 15 and 16, 2018.

\section{Summary}

Percentage change in eGFR, which has been demonstrated to be a potentially useful surrogate endpoint in Europeans and Americans for replacing a hard endpoint of ESRD to indicate the effectiveness of treatments for CKD, was shown to have the same potential usefulness in Japanese.

For both diabetic nephropathy and other CKDs, 30 or $40 \%$ decrease in eGFR over 2 or 3 years may be adopted as surrogate endpoints. However, given that the appropriate observational period and cutoff value of percentage change in eGFR may differ according to the study design (characteristics of subjects, underlying disease, test drug, etc.), there is a need to set appropriate surrogate endpoints when designing and implementing a study.
The evidence for generally using changes in albuminuria/ proteinuria as the surrogate endpoint of ESRD is not sufficient at this point. Depending on the disease, decrease in albuminuria/proteinuria may be used as a surrogate endpoint to replace the prevention of progression to ESRD. However, there is a need to continue accumulating and evaluating the evidence.

The clinical evaluation method for early CKD is also an important task. At this point, the evidence for setting this is insufficient. But clinical evaluation methods applying changes in albuminuria/proteinuria and GFR are drawing interests. This would require studies designed for this purpose and the accumulation of evidence.

\section{Research team (by Japanese alphabetical order, *is the committee leader)}

Kunitoshi Iseki Clinical Research Support Center, Tomishiro Central Hospital, Okinawa, Japan

Kohjiro Ueki Diabetes Research Center, National Center for Global Health and Medicine, Tokyo, Japan

Tomoko Usui Department of Pharmacoepidemiology, Graduate School of Medicine and Public Health, Kyoto University, Kyoto, Japan

Hirokazu Okada Department of Nephrology, Saitama Medical University, Saitama, Japan

Naoki Kashihara Department of Nephrology and Hypertension, Kawasaki Medical School, Okayama, Japan

Eiichiro Kanda Medical Science, Kawasaki Medical School, Okayama, Japan

Tetsuhiro Tanaka Division of Nephrology and Endocrinology, the University of Tokyo, Tokyo, Japan

*Masaomi Nangaku Division of Nephrology and Endocrinology, Graduate School of Medicine, the University of Tokyo, Tokyo, Japan

Kunihiro Matsushita Department of Epidemiology, Johns Hopkins Bloomberg School of Public Health, Maryland, USA

Kenichi Mikami Office of New Drug I, Pharmaceuticals and Medical Devices Agency (PMDA), Tokyo, Japan

Takashi Wada Department of Nephrology and Laboratory Medicine, Kanazawa University, Ishikawa, Japan

Hirotaka Watada Department of Metabolism and Endocrinology, Juntendo University Graduate School of Medicine, Tokyo, Japan 


\section{Systematic review team (by Japanese alphabetical order, *is the working group leader)}

Tomoko Usui Department of Pharmacoepidemiology, Graduate School of Medicine and Public Health, Kyoto University, Kyoto, Japan

*Hirokazu Okada Department of Nephrology, Faculty of Medicine, Saitama Medical University, Saitama, Japan Koji Tomori Department of Nephrology, Faculty of Medicine, Saitama Medical University, Saitama, Japan Yusuke Watanabe Department of Nephrology, Saitama Medical University International Medical Center, Saitama, Japan

Acknowledgements This study was supported by the Research on Regulatory Science of Pharmaceuticals and Medical Devices of AMED. We would like to thank the following for their valuable advice and support in the preparation of this guideline: Toru Kawanishi, Director of National Institute of Health Sciences, Hajime Yasuhara, Managing Director of Showa University Medical Foundation, Takashi Kadowaki, Chairman of the Japan Diabetes Society, Kazuishi Sekino, Kinue Nishioka, Mutsuhiro Ikuma, Hiroyuki Murakami, Kayo Shinohara, Norikazu Hinamoto, Mototsugu Tanaka, Junichi Asano, Tomoko Iwata, Hiroaki Yamada, Ryuta Nakamura, Motoko Honda, Yoshiko Komura, and Takaaki Suzuki of Office of New Drug I/Office of new Drug II, Pharmaceuticals and Medical Devices Agency, Chiho Iseki of Okinawa Heart and Renal Association, Takao Inoue, Reiko Nakajima of Project of Translational and Clinical Research Core Centers AMED, and Masanobu Yamada and Eri Sugiyama of Pharmaceutical Evaluation Division, Pharmaceutical Safety and Environmental Health Bureau, Ministry of Health, Welfare and Labour.

Funding AMED research expenses were used for preparing this guideline. Some of the meeting costs were borne by the Japanese Society of Nephrology and the Japan Diabetes Society. No companies provided funds.

\section{Compliance with ethical standards}

Conflict of interest All the members involved in creating the guidelines submitted conflict of interest statements based on academic society rules, which are managed by Japanese Society of Nephrology. These specific conflict of interest statements described are as follows. Employment: Kunitoshi Iseki, Clinical Research Support Center, Tomishiro Central Hospital, Okinawa, Japan; Kohjiro Ueki, Diabetes Research Center, National Center for Global Health and Medicine, Tokyo, Japan; Tomoko Usui, Department of Pharmacoepidemiology, Graduate School of Medicine and Public Health, Kyoto University, Kyoto, Japan; Hirokazu Okada, Department of Nephrology, Saitama Medical University, Saitama, Japan; Naoki Kashihara, Department of Nephrology and Hypertension, Kawasaki Medical School, Okayama, Japan; Eiichiro Kanda, Medical Science, Kawasaki Medical School, Okayama, Japan; Tetsuhiro Tanaka, Division of Nephrology and Endocrinology, Graduate School of Medicine, the University of Tokyo, Tokyo, Japan; Masaomi Nangaku, Division of Nephrology and Endocrinology, Graduate School of Medicine, the University of Tokyo, Tokyo, Japan; Kunihiro Matsushita, Department of Epidemiology, Johns Hopkins Bloomberg School of Public Health, Maryland, USA; Kenichi Mikami, Office of New Drug I, Pharmaceuticals and Medical
Devices Agency (PMDA), Tokyo, Japan; Takashi Wada, Department of Nephrology and Laboratory Medicine, Kanazawa University, Ishikawa, Japan; Hirotada Watada, Department of Metabolism and Endocrinology, Juntendo University Graduate School of Medicine, Tokyo, Japan. Consultancies: Kunitoshi Iseki, None; Kohjiro Ueki, None; Tomoko Usui, None; Hirokazu Okada, None; Naoki Kashihara, None; Eiichiro Kanda, None; Tetsuhiro Tanaka, None; Masaomi Nangaku, None; Kunihiro Matsushita, None; Kenichi Mikami, None; Takashi Wada, None; Hirotada Watada, None. Honoraria: Kunitoshi Iseki, None; Kohjiro Ueki, MSD K.K., Kyowa Hakko Kirin Co., Ltd., Daiichi Sankyo Ltd., Takeda Pharmaceutical Co., Ltd., Nippon Boehringer Ingelheim Co., Ltd., Novo Nordisk Pharma Ltd., Mitsubishi Tanabe Pharma Corporation, Ono Pharmaceutical Co., Ltd., Eli Lilly Japan K.K.; Tomoko Usui, None; Hirokazu Okada, Alexion Pharmaceuticals, Inc., Kyowa Hakko Kirin Co., Ltd; Naoki Kashihara, Astellas Pharma Inc., MSD K.K., Otsuka Pharmaceutical Co., Ltd., Shionogi \& Co., Ltd., Daiichi Sankyo Ltd., Sumitomo Dainippon Pharma Co., Ltd., Takeda Pharmaceutical Co., Ltd., Nippon Boehringer Ingelheim Co., Ltd., Novartis Pharma K.K; Eiichiro Kanda, None; Tetsuhiro Tanaka, None; Masaomi Nangaku, Astellas Pharma Inc., AstraZeneca K.K., Alexion Pharmaceuticals, Inc., MSD K.K., Kyowa Hakko Kirin Co., Ltd., Daiichi Sankyo Ltd., Mitsubishi Tanabe Pharma Co., Chugai Pharmaceutical Co., Ltd., Japan Tobacco Inc.; Kunihiro Matsushita, Kyowa Hakko Kirin Co., Ltd; Kenichi Mikami, None; Takashi Wada, None; Hirotada Watada, Astellas Pharma, AstraZeneca, Boehringer Ingelheim, Daiichi Sankyo, Dainippon Sumitomo Pharma, Eli Lilly, Kissei Pharma, Kowa, Kyowa Hakko Kirin, Merck Sharp \& Dohme, Mitsubishi Tanabe Pharma, Nippon Boehringer Ingelheim Co., Ltd., Novo Nordisk, Novartis, Ono Pharmaceutical, Sanofi, Sanwa Kagaku Kenkyusho, Takeda Pharmaceutical Co., Ltd. Stock ownership or options: Kunitoshi Iseki, None; Kohjiro Ueki, None; Tomoko Usui, None; Hirokazu Okada, None; Naoki Kashihara, None; Eiichiro Kanda, None; Tetsuhiro Tanaka, None; Masaomi Nangaku, None; Kunihiro Matsushita, None; Kenichi Mikami, None; Takashi Wada, None; Hirotada Watada, None. Grants received: Kunitoshi Iseki, None; Kohjiro Ueki, Astellas Pharma Inc., Kyowa Hakko Kirin Co., Ltd., Daiichi Sankyo Ltd., Takeda, Nippon Boehringer Ingelheim Co., Ltd., Sanofi K.K.; Tomoko Usui, None; Hirokazu Okada, Astellas Pharma Inc., MSD K.K. Kyowa Hakko Kirin Co., Ltd., Daiichi Sankyo Ltd., Takeda Pharmaceutical Co., Ltd., Chugai Pharmaceutical Co., Ltd., Torii Pharmaceutical, Nippon Boehringer Ingelheim Co., Ltd.; Naoki Kashihara, Sanwa Kagaku Kenkyujo Co., Ltd., Fuji Yakuhin Co., Ltd., Astellas Pharma Inc., AstraZeneca K.K., Alexion Pharmaceuticals, Inc., MSD K.K., Otsuka Pharmaceutical Co., Ltd., Kyowa Hakko Kirin Co., Ltd., Daiichi Sankyo Ltd., Sumitomo Dainippon Pharma Co., Ltd., Takeda Pharmaceutical Co., Ltd., Chugai Pharmaceutical Co., Ltd., Teijin Pharma Ltd., Nippon Boehringer Ingelheim Co., Ltd., Bayer Yakuhin, Ltd., Pfizer Japan Inc.; Eiichiro Kanda, None; Tetsuhiro Tanaka, None; Masaomi Nangaku, Astellas Pharma Inc., Alexion Pharmaceuticals, Inc., Kissei Pharmaceutical Co., Ltd., Kyowa Hakko Kirin Co., Ltd., Daiichi Sankyo Ltd., Takeda Pharmaceutical Co., Ltd., Chugai Pharmaceutical Co., Ltd., Nippon Boehringer Ingelheim Co., Ltd. Bayer Yakuhin, Ltd., Mochida Pharmaceutical Co., Ltd.; Kunihiro Matsushita, Kyowa Hakko Kirin Co., Ltd.; Kenichi Mikami, None; Takashi Wada, Shiseido, Kyowa Hakko Kirin Co., Ltd., Sanwa Kagaku Kenkyujo Co., Ltd., Chugai Pharmaceutical Co., Ltd., Takeda Pharmaceutical Co., Ltd., Pfizer Japan Inc.; Hirotada Watada, Abbott, Astellas Pharma, AstraZeneca, Bayer, Benefit One Health Care, Boehringer Ingelheim, Bristol-Myers Squibb, Daiichi Sankyo, Dainippon Sumitomo Pharma, Eli Lilly, Kissei Pharma, Kowa, Kyowa Hakko Kirin, Johnson \& Johnson, Merck Sharp \& Dohme, Mitsubishi Tanabe Pharma, Mochida Pharmaceutical, Nitto Boseki, Novartis, Novo Nordisk, Ono Pharmaceutical, Pfizer, Sanofi, Sanwa Kagaku Kenkyusho, Taisho Toyama Pharmaceutical, Takeda, and Terumo Corp. 
Open Access This article is distributed under the terms of the Creative Commons Attribution 4.0 International License (http://creativeco mmons.org/licenses/by/4.0/), which permits unrestricted use, distribution, and reproduction in any medium, provided you give appropriate credit to the original author(s) and the source, provide a link to the Creative Commons license, and indicate if changes were made.

\section{References}

1. Levin A, Tonelli M, Bonventre J, Coresh J, Donner JA, Fogo AB, Fox CS, Gansevoort RT, Heerspink HJL, Jardine M, Kasiske B, Köttgen A, Kretzler M, Levey AS, Luyckx VA, Mehta R, Moe O, Obrador G, Pannu N, Parikh CR, Perkovic V, Pollock C, Stenvinkel P, Tuttle KR, Wheeler DC, Eckardt KU, ISN Global Kidney Health Summit participants. Global kidney health 2017 and beyond: a roadmap for closing gaps in care, research, and policy. Lancet. 2017;S0140-6736(17)30788-2.

2. Imai E, Horio M, Watanabe T, Iseki K, Yamagata K, Hara S, Ura N, Kiyohara Y, Moriyama T, Ando Y, Fujimoto S, Konta T, Yokoyama H, Makino H, Hishida A, Matsuo S. Prevalence of chronic kidney disease in the Japanese general population. Clin Exp Nephrol. 2009;13:621-30.

3. Palmer SC, Sciancalepore M, Strippoli GF. Trial quality in nephrology: how are we measuring up? Am J Kidney Dis. 2011;58:335-7.

4. Archdeacon P, Shaffer RN, Winkelmayer WC, Falk RJ, RoyChaudhury P. Fostering innovation, advancing patient safety: the kidney health initiative. Clin J Am Soc Nephrol. 2013;8:1609-17.

5. Baigent $\mathrm{C}$, Herrington WG, Coresh J, Landray MJ, Levin A, Perkovic V, Pfeffer MA, Rossing P, Walsh M, Wanner C, Wheeler DC, Winkelmayer WC, McMurray JJV, KDIGO Controversies Conference on Challenges in the Conduct of Clinical Trials in Nephrology Conference Participants. Challenges in conducting clinical trials in nephrology: conclusions from a Kidney DiseaseImproving Global Outcomes (KDIGO) Controversies Conference. Kidney Int. 2017;92:297-305.

6. Biomarkers Definitions Working Group. Biomarkers and surrogate endpoints: preferred definitions and conceptual framework. Clin Pharmacol Ther. 2001;69:89-95.

7. International Conference on Harmonization of Technical Requirements for Registration of Pharmaceuticals for Human Use (ICH E9). Statistical Principles for Clinical Trials. 1998. https://www. pmda.go.jp/files/000156112.pdf.

8. Coresh J, Turin TC, Matsushita K, Sang Y, Ballew SH, Appel LJ, Arima H, Chadban SJ, Cirillo M, Djurdjev O, Green JA, Heine GH, Inker LA, Irie F, Ishani A, Ix JH, Kovesdy CP, Marks A, Ohkubo T, Shalev V, Shankar A, Wen CP, de Jong PE, Iseki K, Stengel B, Gansevoort RT, Levey AS. Decline in estimated glomerular filtration rate and subsequent risk of end-stage renal disease and mortality. JAMA. 2014;311:2518-31.

9. Inker LA, Lambers Heerspink HJ, Mondal H, Mondal H, Schmid $\mathrm{CH}$, Tighiouart H, Noubary F, Coresh J, Greene T, Levey AS. GFR decline as an alternative end point to kidney failure in clinical trials: a meta-analysis of treatment effects from 37 randomized trials. Am J Kidney Dis. 2014;64:848-59.

10. Lambers Heerspink HJ, Tighiouart H, Sang Y, Sang Y, Ballew S, Mondal H, Matsushita K, Coresh J, Levey AS, Inker LA. GFR decline and subsequent risk of established kidney outcomes: a meta-analysis of 37 randomized controlled trials. Am J Kidney Dis. 2014;64:860-6.

11. Greene T, Teng CC, Inker LA, Redd A, Ying J, Woodward M, Coresh J, Levey AS. Utility and validity of estimated GFR-based surrogate time-to-event end points in CKD: a simulation study. Am J Kidney Dis. 2014;64:867-79.

12. Levey AS, Inker LA, Matsushita K, Greene T, Willis K, Lewis E, de Zeeuw D, Cheung AK, Coresh J. GFR decline as an end point for clinical trials in CKD: a scientific workshop sponsored by the National Kidney Foundation and the US Food and Drug Administration. Am J Kidney Dis. 2014;64:821-35.

13. Floege J, Mak RH, Molitoris BA, Remuzzi G, Ronco P. Nephrology research-the past, present and future. Nat Rev Nephrol. 2015;11:677-87.

14. Mol PG, Maciulaitis R, Vetter T. GFR decline as an end point for clinical trials in CKD: a view from Europe. Am J Kidney Dis. 2014;64:838-40.

15. Thompson A, Lawrence J, Stockbridge N. GFR decline as an end point in trials of CKD: a viewpoint from the FDA. Am J Kidney Dis. 2014;64:836-7.

16. Jha V, Garcia-Garcia G, Iseki K, Li Z, Naicker S, Plattner B, Saran $\mathrm{R}$, Wang AY, Yang CW. Chronic kidney disease: global dimension and perspectives. Lancet. 2013;382:260-72.

17. Wen CP, Matsushita K, Coresh J, Iseki K, Islam M, Katz R, McClellan W, Peralta CA, Wang H, de Zeeuw D, Astor BC, Gansevoort RT, Levey AS, Levin A. Chronic kidney disease prognosis consortium. Relative risks of chronic kidney disease for mortality and end-stage renal disease across races are similar. Kidney Int. 2014;86:819-27.

18. Chang WX, Asakawa S, Toyoki D, Nemoto Y, Morimoto C, Tamura Y, Ota T, Shibata S, Fujigaki Y, Shen ZY, Uchida S. Predictors and the subsequent risk of end-stage renal diseaseusefulness of $30 \%$ decline in estimated GFR over 2 years. PLoS One. 2015;10:e132927.

19. Ueki K, Sasako T, Okazaki Y, Kato M, Okahata S, Katsuyama H, Haraguchi M, Morita A, Ohashi K, Hara K, Morise A, Izumi K, Ishizuka N, Ohashi Y, Noda M, Kadowaki T. Effect of intensified multifactorial intervention on cardiovascular outcomes and mortality in patients with type 2 diabetes in J-DOIT3, a multicenter, randomized parallel-group trial. Lancet Diabetes Endocrinol. 2017;5:951-64.

20. Matsushita K, Chen J, Sang Y, Ballew SH, Shimazaki R, Fukagawa M, Imai E, Coresh J, Hishida A. Risk of end-stage renal disease in Japanese patients with chronic kidney disease increases proportionately to decline in estimated glomerular filtration rate. Kidney Int. 2016;90:1109-14.

21. Imai E, Matsuo S, Makino H, Watanabe T, Akizawa T, Nitta K, Iimuro S, Ohashi Y, Hishida A, CKD-JAC Study Group. Chronic kidney disease Japan cohort (CKD-JAC) study: design and methods. Hypertens Res. 2008;31:1101-7.

22. Imai E, Matsuo S, Makino H, Watanabe $\mathrm{T}$, Akizawa $\mathrm{T}$, Nitta $\mathrm{K}$, Iimuro S, Ohashi Y, Hishida A. Chronic Kidney Disease Japan Cohort study: baseline characteristics and factors associated with causative diseases and renal function. Clin Exp Nephrol. 2010;14:558-70.

23. Matsuo S, Imai E, Horio M, Yasuda Y, Tomita K, Nitta K, Yamagata K, Tomino Y, Yokoyama H, Hishida A. Collaborators developing the Japanese equation for estimated GFR. Revised equations for estimated GFR from serum creatinine in Japan. Am J Kidney Dis. 2009;53:982-92.

24. Eriksen BO, Ingebretsen OC. The progression of chronic kidney disease: a 10-year population-based study of the effects of gender and age. Kidney Int. 2006;69:375-82.

25. Neugarten J, Acharya A, Silbiger SR. Effect of gender on the progression of nondiabetic renal disease: a meta-analysis. J Am Soc Nephrol. 2000;11:319-29.

26. Jafar TH, Schmid CH, Stark PC, Toto R, Remuzzi G, Ruggenenti P, Marcantoni C, Becker G, Shahinfar S, De Jong PE, De Zeeuw D, Kamper AL, Strangaard S, Levey AS. The rate of progression 
of renal disease may not be slower in women compared with men: a patient-level meta-analysis. Nephrol Dial Transplant. 2003;18:2047-53.

27. Turin TC, Coresh J, Tonelli M, Stevens PE, de Jong PE, Farmer CK, Matsushita K, Hemmelgarn BR. Short-term change in kidney function and risk of end-stage renal disease. Nephrol Dial Transplant. 2012;27:3835-43.

28. Rebholz CM, Grams ME, Matsushita K, Selvin E, Coresh J. Change in novel filtration markers and risk of ESRD. Am J Kidney Dis. 2015;66:47-54.

29. Kovesdy CP, Coresh J, Ballew SH, Woodward M, Levin A, Naimark DM, Nally J, Rothenbacher D, Stengel B, Iseki K, Matsushita K, Levey AS. CKD prognosis consortium. Past decline versus current eGFR and subsequent ESRD risk. J Am Soc Nephrol. 2016;27:2447-55.

30. Mozaffarian D, Fahimi S, Singh GM, Micha R, Khatibzadeh S, Engell RE, Lim S, Danaei G, Ezzati M, Powles J, Global Burden of Diseases Nutrition and Chronic Diseases Expert Group. Global sodium consumption and death from cardiovascular causes. $\mathrm{N}$ Engl J Med. 2014;371:624-34.

31. Ueshima H, Sekikawa A, Miura K, Turin TC, Takashima N, Kita Y, Watanabe M, Kadota A, Okuda N, Kadowaki T, Nakamura Y, Okamura T. Cardiovascular disease and risk factors in Asia: a selected review. Circulation. 2008;118:2702-9.

32. Iseki K, Kinjo K, Iseki C, Takishita S. Relationship between predicted creatinine clearance and proteinuria and the risk of developing ESRD in Okinawa, Japan. Am J Kidney Dis. 2004;44:806-14.

33. Kanda E, Usui T, Kashihara N, Iseki C, Iseki K, Nangaku M. Importance of glomerular filtration rate change as surrogate endpoint for end-stage renal disease in general Japanese population: community-based cohort study. Clin Exp Nephrol. 2018;22:318-27

34. Imai E, Chan JC, Ito S, Yamasaki T, Kobayashi F, Haneda M, Makino H. ORIENT study investigators. Effects of olmesartan on renal and cardiovascular outcomes in type 2 diabetes with overt nephropathy: a multicentre, randomised, placebo-controlled study. Diabetologia. 2011;54:2978-86.

35. Levey AS, Stevens LA, Schmid CH, Zhang YL, Castro AF, Feldman HI, Kusek JW, Eggers P, Van Lente F, Greene T, Coresh J. CKD-EPI (Chronic Kidney Disease Epidemiology Collaboration). A new equation to estimate glomerular filtration rate. Ann Intern Med. 2009;150:604-12.

36. Inker LA, Lambers Heerspink HJ, Mondal H, et al. GFR decline as an alternative end point to kidney failure in clinical trials: a meta-analysis of treatment effects from 37 randomized trials. Am J Kidney Dis. 2014;64:848-59.

37. Clase CM, Barzilay J, Gao P, Smyth A, Schmieder RE, Tobe S, Teo KK, Yusuf S, Mann JF. Acute change in glomerular filtration rate with inhibition of the renin-angiotensin system does not predict subsequent renal and cardiovascular outcomes. Kidney Int. 2017;91:683-90.

38. Skupien J, Warram JH, Smiles AM, Niewczas MA, Gohda T, Pezzolesi MG, Cantarovich D, Stanton R, Krolewski AS. The early decline in renal function in patients with type 1 diabetes and proteinuria predicts the risk of end-stage renal disease. Kidney Int. 2012;82:589-97.

39. Lambers Heerspink HJ, Weldegiorgis M, Inker LA, Gansevoort R, Parving HH, Dwyer JP, Mondal H, Coresh J, Greene T, Levey AS, de Zeeuw D. Estimated GFR decline as a surrogate end point for kidney failure: a post hoc analysis from the reduction of end points in non-insulin-dependent diabetes with the Angiotensin II Antagonist Losartan (RENAAL) study and Irbesartan Diabetic nephropathy trial (IDNT). Am J Kidney Dis. 2014;63:244-50.

40. Inker LA, Lambers Heerspink HJ, Mondal H, Schmid CH, Tighiouart H, Noubary F, Coresh J, Greene T, Levey AS. GFR decline as an alternative end point to kidney failure in clinical trials: a meta-analysis of treatment effects from 37 randomized trials. Am J Kidney Dis. 2014;64:848-59.

41. Iseki K, Ikemiya Y, Iseki C, Takishita S. Proteinuria and the risk of developing end-stage renal disease. Kidney Int. 2003;63:1468-74.

42. de Zeeuw D, Remuzzi G, Parving HH, Keane WF, Zhang Z, Shahinfar S, Snapinn S, Cooper ME, Mitch WE, Brenner BM. Proteinuria, a target for renoprotection in patients with type 2 diabetic nephropathy: lessons from RENAAL. Kidney Int. 2004;65:2309-20.

43. Atkins RC, Briganti EM, Lewis JB, Hunsicker LG, Braden G, Champion de Crespigny PJ, DeFerrari G, Drury P, Locatelli F, Wiegmann TB, Lewis EJ. Proteinuria reduction and progression to renal failure in patients with type 2 diabetes mellitus and overt nephropathy. Am J Kidney Dis. 2005;45:281-7.

44. Schmieder RE, Mann JF, Schumacher H, Gao P, Mancia G, Weber MA, McQueen M, Koon T, Yusuf S. ONTARGET Investigators. Changes in albuminuria predict mortality and morbidity in patients with vascular disease. J Am Soc Nephrol. 2011;22:1353-64.

45. Inker LA, Levey AS, Pandya K, Stoycheff N, Okparavero A, Greene T. Chronic Kidney Disease Epidemiology Collaboration (CKD-EPI). Early change in proteinuria as a surrogate end point for kidney disease progression: an individual patient meta-analysis. Am J Kidney Dis. 2014;64:74-85.

46. Heerspink HJ, Kröpelin TF, Hoekman J, de Zeeuw D. Reducing albuminuria as surrogate endpoint (REASSURE) consortium. Drug-induced reduction in albuminuria is associated with subsequent renoprotection: a meta-analysis. J Am Soc Nephrol. 2015;26:2055-64.

47. Cravedi P, Ruggenenti P, Remuzzi G. Proteinuria should be used as a surrogate in CKD. Nat Rev Nephrol. 2012;8:301-6.

48. Lambers Heerspink HJ, Gansevoort RT. Albuminuria is an appropriate therapeutic target in patients with CKD: the Pro View. Clin J Am Soc Nephrol. 2015;10:1079-88.

49. Fried LF, Lewis J. Albuminuria is not an appropriate therapeutic target in patients with CKD: the Con View. Clin J Am Soc Nephrol. 2015;10:1089-93.

50. Levey AS, Cattran D, Friedman A, Miller WG, Sedor J, Tuttle K, Kasiske B, Hostetter T. Proteinuria as a surrogate outcome in CKD: report of a scientific workshop sponsored by the National Kidney Foundation and the US Food and Drug Administration. Am J Kidney Dis. 2009;54:205-26.

51. Imai E, Haneda M, Chan JC, Yamasaki T, Kobayashi F, Ito S, Makino H. Reduction and residual proteinuria are therapeutic targets in type 2 diabetes with overt nephropathy: a post hoc analysis (ORIENT-proteinuria). Nephrol Dial Transplant. 2013;28:2526-34.

52. Heerspink HJ, Ninomiya T, Persson F, Brenner BM, Brunel P, Chaturvedi N, Desai AS, Haffner SM, Mcmurray JJ, Solomon $\mathrm{SD}$, Pfeffer MA, Parving HH, de Zeeuw D. Is a reduction in albuminuria associated with renal and cardiovascular protection? A post hoc analysis of the ALTITUDE trial. Diabetes Obes Metab. 2016; 18:169-77.

53. Felix Kröpelin T, de Zeeuw D, Holtkamp FA, Packham DK, L Heerspink HJ. Individual long-term albuminuria exposure during angiotensin receptor blocker therapy is the optimal predictor for renal outcome. Nephrol Dial Transplant. 2016;31:1471-7.

54. Inker LA, Mondal H, Greene T, Masaschi T, Locatelli F, Schena FP, Katafuchi R, Appel GB, Maes BD, Li PK, Praga M, Del Vecchio L, Andrulli S, Manno C, Gutierrez E, Mercer A, Carroll KJ, Schmid CH, Levey AS. Early change in urine protein as a surrogate end point in studies of IgA nephropathy: an individual-patient meta-analysis. Am J Kidney Dis. 2016;68:392-401. 
55. Jun M, Turin TC, Woodward M, Perkovic V, Lambers Heerspink HJ, Manns BJ, Tonelli M, Hemmelgarn BR. Assessing the validity of surrogate outcomes for ESRD: a meta-analysis. J Am Soc Nephrol. 2015;26:2289-302.
56. Usui T, Kanda E, Iseki C, Iseki K, Kashihara N, Nangaku M. Observational period for changes in proteinuria and risk prediction of end-stage renal disease in general population. Nephrology (Carlton). 2017. https://doi.org/10.1111/nep.13093. 